ETH-TH/95-42

SLAC-PUB-95-7073

December 1995

\title{
Three-jet cross sections to next-to-leading order
}

\author{
S. Frixione ${ }^{a}$, Z. Kunszt \\ Theoretical Physics, ETH, Zurich, Switzerland \\ A. Signer ${ }^{a}$ \\ SLAC, PO Box 4349, Stanford, CA 94309
}

\begin{abstract}
One- and two-jet inclusive quantities in hadron collisions have already been calculated to next-to-leading order accuracy, using both the subtraction and the cone method. Since the one-loop corrections have recently been obtained for all five-parton amplitudes, three-jet inclusive quantities can also be predicted to next-to-leading order. The subtraction method presented in the literature is based on a systematic use of boost-invariant kinematical variables, and therefore its application to three-jet production is quite cumbersome. In this paper we reanalyze the subtraction method and point out the advantage of using angle and energy variables. This leads to simpler results and it has complete generality, extending its validity to $n$-jet production. The formalism is also applicable to $n$-jet production in $e^{+} e^{-}$annihilation and in photon-hadron collisions. All the analytical results necessary to construct an efficient numerical program for next-to-leading order three-jet inclusive quantities in hadroproduction are given explicitly. As new analytical result, we also report the collinear limits of all the two-to-four processes.
\end{abstract}

${ }^{a}$ Work supported by the National Swiss Foundation 


\section{Introduction}

The production of one or more jets is an important phenomenon in large transverse momentum reactions at hadron-hadron and photon-hadron colliders [1]. Jet production has large rates and rich final state structure, thus providing an excellent testing ground for the predictions of perturbative QCD and a formidable background to various new physics signals. It is therefore important to have as significant theoretical predictions as possible for all the measured distributions in jet production.

In the last decade the formalism of the next-to-leading order perturbative QCD has successfully been applied [2-44 to explain quantitatively the data on one-jet and two-jet inclusive quantities obtained at SPS, TEVATRON and HERA [5]. At the next-toleading order the theoretical uncertainties can be reduced below the experimental errors and impressive agreement has been found between the data and the theoretical predictions for all available inclusive one-jet or two-jet measurements.

We recall, however, that there are two embarrassing unresolved discrepancies. First, the ramping run at TEVATRON could be used to test Feynman scaling; the data show larger scaling violation effects than predicted by the theory [5]. Second, the tail of the transverse energy distribution of single-inclusive jet production, measured by the CDF collaboration [6], appears to be higher than the next-to-leading order QCD prediction (see, however, ref. [10).

The success (and also the possible difficulties) of the next-to-leading order description of one- and two-jet production, and the large number of three-jet events collected by experimental collaborations [11], motivate the extension of the quantitative next-to-leading order analysis also to the description of the three-jet data. The study of the ratio of three- to two-jet production rates is expected to give a clean measurement of $\alpha_{S}$ at hadron colliders.

The theoretical analysis of inclusive three-jet production is rather complex. The five-parton amplitudes have to be calculated to next-to-leading order. As a result of significant technical developments based on the helicity method, supersymmetry and string theory, these calculations have been performed and presently the theoretical inputs at the amplitude level are fully available 12-15. The leading order six-parton amplitudes, which have been obtained long time ago [16 18], are also needed. The relation of the physical cross section with the partonic amplitudes, however, is rather involved, since the soft and collinear singularities appearing in the next-to-leading 
order amplitude of the $2 \rightarrow 3$ parton scattering and in the leading-order $2 \rightarrow 4$ amplitudes are cancelled only when the cross section of inclusive, infrared-safe quantities is considered. In an efficient phenomenological numerical study, the soft and collinear singularities appearing in the loop corrections and in the real contributions have to be cancelled analytically. It has been pointed out in refs. [19-21 that the necessary analytical cancellation can always be achieved due to good universal properties of the soft and collinear limiting behavior of partonic amplitudes. In particular, two methods have been proposed: the so-called subtraction method [19,20] and the cone method [21]. Both formalisms have successfully been applied to describe various distributions measured in inclusive one-jet and two-jet production.

The aim of this paper is to present some further improvements of the formalism of the subtraction method necessary for the phenomenological study of three-jet inclusive quantities. The subtraction method of ref. [19] is based on a systematic use of boost-invariant kinematical variables when the reference to the beam direction remains always explicit. As a result, the soft integrals become more complicated than their inherent structure would require and their treatment is somewhat cumbersome when it is applied to three or more jet production.

We point out that the formalism of ref. [19] remains valid also when energy and angle variables are used. In this case the soft integrals become much simpler and the formalism is generally valid for inclusive production of any number of jets. The subtraction method with energy and angle variables has already been applied to the description of other production processes; for a formulation quite close to the one we use in this paper, see ref. [22].

The cancellation of leading singularities does not completely solve the numerical problems. The subtracted cross sections may still have integrable square-root singularities, thus resulting in an inefficient numerical evaluation. In ref. [19] this difficulty has been overcome by decomposing the cross section with partial fractioning into terms of single-singular factors. This method is not practical in the case of three-jet production since it leads to very long algebraic formulae. We shall show that such a decomposition is not actually necessary, since the measurement function defining infrared-safe inclusive jet quantities automatically splits the squared amplitude into terms where only one (or at most two) singular regions can contribute.

In this paper we summarize the results obtained for the next-to-leading order fiveparton amplitudes, we construct local subtraction terms for the singular regions of the 
six-parton cross sections and we calculate the contributions of the soft and collinear regions analytically. With the latter result we have all the analytic formulae at our disposal to construct an efficient numerical program for the evaluation of three-jet production rates to next-to-leading order accuracy.

Our paper is organized as follows. In section 2 the organization of the calculation is presented: our definitions and conventions are given, the $\overline{\mathrm{MS}}$ collinear counterterms are explicitly worked out, the adopted jet-finding algorithm is described and the measurement functions are constructed. In section 3 we remind the known results for the virtual contribution. In section 4 we deal with the real contribution, and we show how to decompose it into single-singular factors. We define the soft subtraction using angle and energy variables and we evaluate the soft integrals analytically. We next turn to the definition of the subtraction terms for the initial and final state collinear singularities; all the needed integrals are analytically calculated. In section 5 we collect our results, and we show that when we sum the contributions of the real term, of the collinear counterterms and of the virtual term all the singularities cancel. The numerical implementation of our analytical results is also shortly discussed. Section 6 contains our concluding remarks. The soft and collinear integrals are collected in appendix A. Finally, in appendix B we present a detailed description of the collinear limits of the $n$-parton cross sections.

\section{The jet cross section}

\subsection{Introductory remarks}

Thanks to the factorization theorem [23], a generic differential cross section in hadronic collisions can be written in the following way

$$
d \sigma^{\left(H_{1} H_{2}\right)}\left(K_{1}, K_{2}\right)=\sum_{a b} \int d x_{1} d x_{2} f_{a}^{\left(H_{1}\right)}\left(x_{1}\right) f_{b}^{\left(H_{2}\right)}\left(x_{2}\right) d \hat{\sigma}_{a b}\left(x_{1} K_{1}, x_{2} K_{2}\right)
$$

where $H_{1}$ and $H_{2}$ are the incoming hadrons, $K_{1}$ and $K_{2}$ their momentum, and the sum runs over all the parton flavours which give a non-trivial contribution. The quantities $d \hat{\sigma}_{a b}$ are the subtracted partonic cross sections, in which the singularities due to collinear emission of massless partons from the incoming partons have been cancelled by some suitable counterterms.

We first express the subtracted cross sections in terms of the unsubtracted ones, 
which can be directly calculated in perturbative QCD. To this end, we have to write the collinear counterterms. Due to universality, eq. (2.1) applies also when the incoming hadrons are formally substituted with partons. In this case, we are also able to evaluate the partonic densities, which at the next-to-leading order read

$$
f_{a}^{(d)}(x)=\delta_{a d} \delta(1-x)-\frac{\alpha_{S}}{2 \pi}\left(\frac{1}{\bar{\epsilon}} P_{a d}(x, 0)-K_{a d}(x)\right)+\mathcal{O}\left(\alpha_{S}^{2}\right),
$$

where $P_{a d}(x, 0)$ are the Altarelli-Parisi kernels in four dimensions (since we will usually work in $4-2 \epsilon$ dimensions, the 0 in the argument of $P_{a d}$ stands for $\epsilon=0$ ) and the functions $K_{a d}$ depend upon the subtraction scheme in which the calculation is carried out. For $\overline{\mathrm{MS}}, K_{a d} \equiv 0$. Writing the perturbative expansion of the unsubtracted and subtracted partonic cross sections at next-to-leading order as

$$
d \sigma_{a b}=d \sigma_{a b}^{(0)}+d \sigma_{a b}^{(1)}, \quad d \hat{\sigma}_{a b}=d \hat{\sigma}_{a b}^{(0)}+d \hat{\sigma}_{a b}^{(1)}
$$

where the superscript 0 (1) denotes the leading (next-to-leading) order contribution, we have

$$
\begin{aligned}
d \hat{\sigma}_{a b}^{(0)}\left(k_{1}, k_{2}\right)= & d \sigma_{a b}^{(0)}\left(k_{1}, k_{2}\right) \\
d \hat{\sigma}_{a b}^{(1)}\left(k_{1}, k_{2}\right)= & d \sigma_{a b}^{(1)}\left(k_{1}, k_{2}\right)+\frac{\alpha_{S}}{2 \pi} \sum_{d} \int d x\left(\frac{1}{\bar{\epsilon}} P_{d a}(x, 0)-K_{d a}(x)\right) d \sigma_{d b}^{(0)}\left(x k_{1}, k_{2}\right) \\
& +\frac{\alpha_{S}}{2 \pi} \sum_{d} \int d x\left(\frac{1}{\bar{\epsilon}} P_{d b}(x, 0)-K_{d b}(x)\right) d \sigma_{a d}^{(0)}\left(k_{1}, x k_{2}\right) .
\end{aligned}
$$

The second and the third term in the RHS of eq. (2.5) are the collinear counterterms we were looking for. Notice that in this equation the Born terms $d \sigma^{(0)}$ are evaluated in $4-2 \epsilon$ dimensions.

For three-jet production, the leading-order cross section can get contributions only from the two-to-three partonic subprocesses. We write this contribution in the following way

$$
\begin{aligned}
d \sigma_{a_{1} a_{2}}^{(0)}\left(k_{1}, k_{2} ;\left\{J_{l}\right\}_{1,3}\right) & =\frac{1}{3 !} \sum_{\left\{a_{l}\right\}_{3,5}} \mathcal{M}^{(3,0)}\left(\left\{a_{l}\right\}_{1,5} ;\left\{k_{l}\right\}_{1,5}\right) \\
& \times \mathcal{S}_{3}\left(\left\{k_{l}\right\}_{3,5} ;\left\{J_{l}\right\}_{1,3}\right) d \phi_{3}\left(k_{1}, k_{2} \rightarrow\left\{k_{l}\right\}_{3,5}\right) .
\end{aligned}
$$

Here we denoted with

$$
\left\{J_{l}\right\}_{1,3}=\left\{J_{1}, J_{2}, J_{3}\right\}
$$


the set of the four-momenta of the jets. In the following, we will almost always suppress the indication of the $\left\{J_{l}\right\}_{1,3}$ dependence. We have indicated with $k_{i}$ and $a_{i}$ respectively the momentum and the flavour of the parton number $i$ involved in the process; by definition, partons 1 and 2 are the incoming ones. In the sum $\sum_{\left\{a_{l}\right\}_{3,5}}$ every $a_{l}$, with $3 \leq l \leq 5$, takes the values $g, u, \bar{u}$, and so on. To avoid overcounting in physical predictions, we inserted the factor $1 / 3$ !. To shorten as much as possible the notation, we have collectively indicated the momenta as

$$
\left\{k_{l}\right\}_{i, j} \equiv\left\{k_{l} \mid i \leq l \leq j\right\} .
$$

It will also turn useful to define

$$
\left\{k_{l}\right\}_{i, j}^{[n p . .]} \equiv\left\{k_{l} \mid i \leq l \leq j, l \neq n, l \neq p, . .\right\} .
$$

The same notation will be used for flavours. The quantity $\mathcal{S}_{3}$ is the so-called measurement function, which defines the infrared-safe jet observables in terms of the momenta of the (unobservable) partons; we will describe it in more details in the following. $\mathcal{M}^{(3,0)}$ is the two-to-three leading-order transition amplitude squared, summed over final state and averaged over initial state color and spin degrees of freedom, and multiplied by the flux factor

$$
\mathcal{M}^{(3,0)}=\frac{1}{2 k_{1} \cdot k_{2}} \frac{1}{\omega\left(a_{1}\right) \omega\left(a_{2}\right)} \sum_{\substack{\text { color } \\ \text { spin }}}\left|\mathcal{A}^{(\text {tree })}(2 \rightarrow 3)\right|^{2}
$$

where $\omega(a)$ is the number of color and spin degrees of freedom for the flavour $a$. We remember that, in $4-2 \epsilon$ dimensions,

$$
\omega(q)=2 N_{c}, \quad \omega(g)=2(1-\epsilon) D_{A},
$$

where $D_{A}=N_{c}^{2}-1$ is the dimension of the adjoint representation of the color group $S U\left(N_{c}\right)$. Since the incoming partons can play a very special rôle, we will also write the functional dependence of $\mathcal{M}^{(3,0)}$ in the following way

$$
\mathcal{M}^{(3,0)}\left(a_{1}, a_{2},\left\{a_{l}\right\}_{3,5} ; k_{1}, k_{2},\left\{k_{l}\right\}_{3,5}\right) \text {. }
$$

The transition amplitude for processes in which only gluons and quarks are involved is usually evaluated in the unphysical configuration in which all the particles are outgoing. The amplitude $\mathcal{A}^{\text {(tree) }}(2 \rightarrow 3)$ of eq. (2.10) can be obtained from the amplitude

$$
\mathcal{A}^{(\text {tree })}(0 \rightarrow 5)=\mathcal{A}^{(\text {tree })}\left(\bar{a}_{1}, \bar{a}_{2},\left\{a_{l}\right\}_{3,5} ;-k_{1},-k_{2},\left\{k_{l}\right\}_{3,5}\right)
$$


simply by crossing (for details on crossing, see appendix B); notice that eq. (2.13) is crossing invariant. Finally, in eq. (2.6) we denoted with $d \phi_{3}$ the full (i.e., with the $\delta$ that enforces the conservation of four-momentum) three-body phase space.

Coming to the next-to-leading order contribution, both the two-to-three and twoto-four partonic subprocesses have to be considered. As customary in perturbative QCD, we denote as virtual the contribution of the former, and as real the contribution of the latter:

$$
d \sigma_{a_{1} a_{2}}^{(1)}=d \sigma_{a_{1} a_{2}}^{(v)}+d \sigma_{a_{1} a_{2}}^{(r)}
$$

with

$$
\begin{aligned}
d \sigma_{a_{1} a_{2}}^{(v)}\left(k_{1}, k_{2} ;\left\{J_{l}\right\}_{1,3}\right) & =\frac{1}{3 !} \sum_{\left\{a_{l}\right\}_{3,5}} \mathcal{M}^{(3,1)}\left(\left\{a_{l}\right\}_{1,5} ;\left\{k_{l}\right\}_{1,5}\right) \\
& \times \mathcal{S}_{3}\left(\left\{k_{l}\right\}_{3,5} ;\left\{J_{l}\right\}_{1,3}\right) d \phi_{3}\left(k_{1}, k_{2} \rightarrow\left\{k_{l}\right\}_{3,5}\right), \\
d \sigma_{a_{1} a_{2}}^{(r)}\left(k_{1}, k_{2} ;\left\{J_{l}\right\}_{1,3}\right) & =\frac{1}{4 !} \sum_{\left\{a_{l}\right\}_{3,6}} \mathcal{M}^{(4)}\left(\left\{a_{l}\right\}_{1,6} ;\left\{k_{l}\right\}_{1,6}\right) \\
& \times \mathcal{S}_{4}\left(\left\{k_{l}\right\}_{3,6} ;\left\{J_{l}\right\}_{1,3}\right) d \phi_{4}\left(k_{1}, k_{2} \rightarrow\left\{k_{l}\right\}_{3,6}\right),
\end{aligned}
$$

where $\mathcal{S}_{4}$ is the measurement function, analogous to $\mathcal{S}_{3}$, for four partons in the final state. $\mathcal{M}^{(3,1)}$ is due to the loop contribution to the two-to-three subprocesses

$$
\begin{aligned}
\mathcal{M}^{(3,1)} & =\frac{1}{2 k_{1} \cdot k_{2}} \frac{1}{\omega\left(a_{1}\right) \omega\left(a_{2}\right)} \\
& \times \sum_{\substack{\text { color } \\
\text { spin }}}\left[\mathcal{A}^{(\text {tree })}(2 \rightarrow 3)\left(\mathcal{A}^{(\text {loop })}(2 \rightarrow 3)\right)^{*}+\left(\mathcal{A}^{(\text {tree })}(2 \rightarrow 3)\right)^{*} \mathcal{A}^{(\text {loop })}(2 \rightarrow 3)\right],
\end{aligned}
$$

while $\mathcal{M}^{(4)}$ is defined in terms of the two-to-four transition amplitude

$$
\mathcal{M}^{(4)}=\frac{1}{2 k_{1} \cdot k_{2}} \frac{1}{\omega\left(a_{1}\right) \omega\left(a_{2}\right)} \sum_{\substack{\text { color } \\ \text { spin }}}\left|\mathcal{A}^{(\text {tree })}(2 \rightarrow 4)\right|^{2}
$$

Notice that in eq. (2.16) the dependence upon the momentum and flavour of the additional parton was inserted. Also, the measurement function, as well as the combinatorial factor $1 / 4$ !, had to be modified with respect to eq. (2.6) and eq. (2.15). The full four-body phase space was denoted with $d \phi_{4}$. 
We can now go back to eq. (2.5), to write explicitly the collinear counterterms for the three-jet production. Using eq. (2.6) we get

$$
\begin{aligned}
d \sigma_{a_{1} a_{2}}^{(c n t+)} & =\frac{1}{3 !} \frac{\alpha_{S}}{2 \pi} \sum_{d} \int d x\left(\frac{1}{\bar{\epsilon}} P_{d a_{1}}(x, 0)-K_{d a_{1}}(x)\right) \\
& \times \sum_{\left\{a_{l}\right\}_{3,5}} \mathcal{M}^{(3,0)}\left(d, a_{2},\left\{a_{l}\right\}_{3,5} ; x k_{1}, k_{2},\left\{k_{l}\right\}_{3,5}\right) \mathcal{S}_{3} d \phi_{3}\left(x k_{1}, k_{2} \rightarrow\left\{k_{l}\right\}_{3,5}\right), \\
d \sigma_{a_{1} a_{2}}^{(c n t-)} & =\frac{1}{3 !} \frac{\alpha_{S}}{2 \pi} \sum_{d} \int d x\left(\frac{1}{\bar{\epsilon}} P_{d a_{2}}(x, 0)-K_{d a_{2}}(x)\right) \\
& \times \sum_{\left\{a_{l}\right\}_{3,5}} \mathcal{M}^{(3,0)}\left(a_{1}, d,\left\{a_{l}\right\}_{3,5} ; k_{1}, x k_{2},\left\{k_{l}\right\}_{3,5}\right) \mathcal{S}_{3} d \phi_{3}\left(k_{1}, x k_{2} \rightarrow\left\{k_{l}\right\}_{3,5}\right) .
\end{aligned}
$$

By construction, these quantities, when added to the unsubtracted three-jet partonic cross section, must cancel the collinear singularities coming from initial state emission.

\subsection{The jet-finding algorithm}

We now turn to the problem of the definition of the measurement functions $\mathcal{S}_{3}$ and $\mathcal{S}_{4}$. As a preliminary, we need some prescription defining the way in which unobservable partons are eventually merged into physical jets. To define a jet in terms of partons, it is customary to distinguish two separate steps: the clustering algorithm, which

decides whether a given set of partons is mergeable into a jet, and the merging procedure, which defines the jet momentum as a function of the parton momenta. The clustering algorithm we choose to use was introduced by Ellis and Soper [24]. It is a $k_{T}$ algorithm specifically designed for hadron-hadron collisions. It is formulated in terms of the transverse momenta $k_{i T}$ and of the lego plot distances $R_{i j}$ of the final state partons

$$
\begin{aligned}
d_{i} & =k_{i T}^{2}, \\
R_{i j} & =\left(\eta_{i}-\eta_{j}\right)^{2}+\left(\varphi_{i}-\varphi_{j}\right)^{2}, \\
d_{i j} & =\min \left(k_{i T}^{2}, k_{j T}^{2}\right) \frac{R_{i j}}{D^{2}},
\end{aligned}
$$

where the constant $D$ is the jet-resolution parameter which value is set at convenience; in practice, $0.4<D<1.0$. The algorithm is defined by means of an iterative 
procedure. One starts with an empty list of jets and a list of protojets, the latter being in the first step by definition identical to the partons. Then, the quantities $d_{i}$ and $d_{i j}$ are evaluated for all the protojets and the minimum among them is found. If this minimum is $d_{i}$, then protojet $i$ is moved from the list of protojets to the list of jets. Otherwise, if the minimum is $d_{i j}$, then protojets $i$ and $j$ are merged into a protojet. The four-momentum of the protojet is defined by means of the merging procedure of ref. [25]: it is the sum of the four-momenta of the two constituent protojets. The jet-finding procedure is repeated as long as there are protojets around. When the list

of jets is completed, that is, the list of protojets is empty, all jets with $k_{T}$ below a certain threshold are dropped. We choose the threshold to be a given fraction (which we denote by $f$ ) of the greatest squared transverse momentum; this means that the threshold has to be recomputed for every event.

Other jet-finding algorithms are obviously possible [2,26]. Nevertheless, preliminary studies 25] indicate that, contrary to $e^{+} e^{-}$annihilation, in hadroproduction the prescription described here is favoured by the data.

\subsection{The measurement function}

Using the jet-finding algorithm of the previous section, we can explicitly define the measurement functions we need to construct the jet cross section.

When there are only three partons in the final state, no merging is possible, and the partons themselves will eventually result in physical jets. Therefore

$$
\begin{aligned}
\mathcal{S}_{3}= & \sum_{\sigma(J)} \delta(j, k, l) \theta\left(\min \left(d_{j}, d_{k}, d_{l}\right)-f \max \left(d_{j}, d_{k}, d_{l}\right)\right) \\
& \times \theta\left(R_{j k}-D^{2}\right) \theta\left(R_{j l}-D^{2}\right) \theta\left(R_{k l}-D^{2}\right),
\end{aligned}
$$

where we introduced the shorthand notation for the $\delta$ over four-momenta

$$
\delta(j, k, l)=\delta\left(k_{j}-J_{1}\right) \delta\left(k_{k}-J_{2}\right) \delta\left(k_{l}-J_{3}\right)
$$

The indices $j, k$ and $l$ take the values 3, 4, 5 and are different from each other. $\sigma(J)$ denotes the permutation over the jet four-momenta $\left\{J_{l}\right\}_{1,3}$.

When four partons are present in the final state, the situation is somewhat more involved. To get three jets starting from four partons, only the following possibilities may occur: 
- no merging, but one parton is dropped being below the hard scale (this contribution will be denoted by $\mathcal{S}_{i}^{(0)}$ );

- one merging, occurring in the first step of the algorithm $\left(\mathcal{S}_{i j}^{(1)}\right)$;

- one merging, occurring in the second step of the algorithm $\left(\mathcal{S}_{i}^{(2)}\right)$;

- one merging, occurring in the third step of the algorithm $\left(\mathcal{S}_{i}^{(3)}\right)$.

Consistently, we will then write

$$
\mathcal{S}_{4}=\sum_{i}\left(\mathcal{S}_{i}^{(0)}+\mathcal{S}_{i}^{(2)}+\mathcal{S}_{i}^{(3)}\right)+\sum_{\substack{i, j \\ i<j}} \mathcal{S}_{i j}^{(1)},
$$

where

$$
\begin{aligned}
\mathcal{S}_{i}^{(0)} & =\sum_{\sigma(J)} \delta(j, k, l) \mathcal{F}_{j k l}^{(i, 0)}, \\
\mathcal{S}_{i j}^{(1)} & =\sum_{\sigma(J)} \delta(i+j, k, l) \mathcal{F}_{k l}^{(i j, 1)}, \\
\mathcal{S}_{i}^{(2)} & =\sum_{\sigma\left(J_{I}\right)} \sum_{\substack{j, k, l \\
k<l}} \delta(i, j, k+l) \mathcal{F}_{j k l}^{(i, 2)}, \\
\mathcal{S}_{i}^{(3)} & =\sum_{\sigma\left(J_{I}\right)} \sum_{\substack{j, k, l \\
k<l}} \delta(i, j, k+l) \mathcal{F}_{j k l}^{(i, 3)},
\end{aligned}
$$

and the indices $j, k$, and $l$ can take the values 3, 4, 5, 6 being different from each other and from $i$

$$
\{j, k, l\}=\{3,4,5,6\} \backslash\{i\} .
$$

To write explicitly the $\mathcal{F}$ functions we introduce the following shorthand definitions

$$
\begin{aligned}
m(. ., i, . ., j+p, . ., m n, . .) & =\min \left(. ., d_{i}, . ., d_{j+p}, . ., d_{m n}, . .\right) \\
M(. ., i, . ., j+p, . ., m n, . . & =\max \left(. ., d_{i}, . ., d_{j+p}, . ., d_{m n}, . .\right)
\end{aligned}
$$

and, consistently, we denote everywhere $d_{i} \equiv i, d_{i j} \equiv i j$. We also write for short

$$
m([a])=m(i, . ., j k, . .) \quad \forall i \neq a, \quad \forall j k \neq a,
$$

where $d_{a}$ can be either $d_{\alpha}$ or $d_{\alpha \beta}$, and the minimum on the RHS is evaluated over the list of all the $d_{i}$ and $d_{j k}$ with the exclusion of $d_{a}$. Finally, we denote

$$
m_{\{a, . ., b, . .\}}([c]),
$$


which is analogous to eq. (2.33); the $\{a, . ., b, .$.$\} inserted means that the list of d_{i}$ and $d_{i j}$ over which the minimum is evaluated is such that it does not contain the indices $a$ and $b$. We then have

$$
\begin{aligned}
\mathcal{F}_{j k l}^{(i, 0)} & =\theta(m([i])-i) \theta(f M(j, k, l)-i) \theta(m(j, k, l)-f M(j, k, l)) \\
& \times \theta\left(R_{j k}-D^{2}\right) \theta\left(R_{j l}-D^{2}\right) \theta\left(R_{k l}-D^{2}\right) \\
\mathcal{F}_{k l}^{(i j, 1)} & =\theta(m([i j])-i j) \theta(m(i+j, k, l)-f M(i+j, k, l)) \\
& \times \theta\left(R_{i+j, k}-D^{2}\right) \theta\left(R_{i+j, l}-D^{2}\right) \theta\left(R_{k l}-D^{2}\right) \\
\mathcal{F}_{j k l}^{(i, 2)} & =\theta(m([i])-i) \theta(m(i, j, k+l)-f M(i, j, k+l)) \\
& \times \theta\left(m_{\{i\}}([k l])-k l\right) \theta\left(R_{k+l, j}-D^{2}\right) \\
\mathcal{F}_{j k l}^{(i, 3)} & =\theta(m([i])-i) \theta(m(i, j, k+l)-f M(i, j, k+l)) \\
& \times \theta\left(m_{\{i\}}([j])-j\right) \theta\left(D^{2}-R_{k l}\right) .
\end{aligned}
$$

\subsection{Infrared singular regions}

It is well known that the perturbatively calculated QCD cross sections, even after the ultraviolet renormalization, have a divergent behaviour, arising from the regions in which a parton (either virtual, that is, exchanged in a loop, or real, that is, emitted and contributing to the final state kinematics) is soft or collinear to another parton. In this section, we will investigate the behaviour of the measurement function in this regions (which we will denote as infrared singular regions) in the case when there are four partons in the final state. The singular regions eventually occurring are as follows

- parton $i(i=3,4,5,6)$ is $\operatorname{soft}\left(k_{i}^{0} \rightarrow 0\right)$;

- parton $i$ is collinear to the incoming parton 1 or $2(i \| 1$ or $i \| 2)$;

- parton $i$ is collinear to parton $j(i \| j)$.

From eqs. (2.35)-(2.38), it is quite easy to prove that the $\mathcal{F}$ functions are non vanishing only in the following singular regions

$$
\begin{aligned}
& \mathcal{F}_{j k l}^{(i, 0)} \quad \Rightarrow \quad k_{i}^{0} \rightarrow 0, \quad i\|1, \quad i\| 2, \\
& \mathcal{F}_{k l}^{(i j, 1)} \quad \Rightarrow \quad k_{i}^{0} \rightarrow 0, \quad k_{j}^{0} \rightarrow 0, \quad i \| j,
\end{aligned}
$$




$$
\begin{array}{ll}
\mathcal{F}_{j k l}^{(i, 2)} & \Rightarrow \text { none }, \\
\mathcal{F}_{j k l}^{(i, 3)} & \Rightarrow \text { none } .
\end{array}
$$

Eq. (2.40) suggests the following decomposition

$$
\mathcal{S}_{i j}^{(1)}=\mathcal{S}_{i j}^{(1)} \theta\left(d_{j}-d_{i}\right)+\mathcal{S}_{i j}^{(1)} \theta\left(d_{i}-d_{j}\right)
$$

in this way, the first term in the RHS of eq. (2.43) does not get contributions when $j$ is soft, and the second one when $i$ is soft. This in turn implies that, after some algebra, we can cast eq. (2.26) in the following form

$$
\mathcal{S}_{4}=\sum_{i}\left(\mathcal{S}_{i}^{(\text {sing })}+\mathcal{S}_{i}^{(\text {fin })}\right)
$$

where

$$
\begin{aligned}
\mathcal{S}_{i}^{(\text {sing })} & =\mathcal{S}_{i}^{(0)}+\sum_{j}^{[i]} \mathcal{S}_{i j}^{(1)} \theta\left(d_{j}-d_{i}\right) \\
\mathcal{S}_{i}^{(f i n)} & =\mathcal{S}_{i}^{(2)}+\mathcal{S}_{i}^{(3)}
\end{aligned}
$$

and the $[i]$ in the sum means that $j$ can take the values $3,4,5,6$ with the exclusion of $i$. The quantity $\mathcal{S}_{i}^{(f i n)}$ does not get any contribution from the singular regions; on the other hand, $\mathcal{S}_{i}^{(\operatorname{sing})}$ is different from 0 when $i$ is soft (or $i\|1, i\| 2$ ), but it is equal to zero when any other parton is soft (or collinear to the incoming partons), thanks to the factor $\theta\left(d_{j}-d_{i}\right)$. The region in which $i \| j$ contributes to $\mathcal{S}_{i}^{(s i n g)}$ and to $\mathcal{S}_{j}^{(s i n g)}$, but the $\theta\left(d_{j}-d_{i}\right)$ inserted in eq. (2.45) prevents any double counting.

We can finally investigate the form of the limiting behaviour of the measurement function $\mathcal{S}_{4}$ in the infrared singular regions.

- $i$ is soft. From eqs. (2.39)-(2.42) and eq. (2.44), we have

$$
\lim _{k_{i}^{0} \rightarrow 0} \mathcal{S}_{4}=\lim _{k_{i}^{0} \rightarrow 0}\left[\mathcal{S}_{i}^{(0)}+\sum_{j}^{[i]} \mathcal{S}_{i j}^{(1)}\right] .
$$

The limit of the $\mathcal{F}$ functions can be very easily evaluated from their definition; it simply amounts to make the formal substitution $i+j \rightarrow j$ wherever $i+j$ appears. We still need to do some combinatorial algebra to get the limit of the full $\mathcal{S}_{4}$; after writing explicitly the terms contributing to the sum and using

$$
\lim _{k_{i}^{0} \rightarrow 0}\left[\theta(m([i])-i)+\sum_{j}^{[i]} \theta(m([i j])-i j)\right]=1
$$


we get

$$
\lim _{k_{i}^{0} \rightarrow 0} \mathcal{S}_{4}=\mathcal{S}_{3}([i])
$$

where

$$
\begin{aligned}
\mathcal{S}_{3}([i])= & \sum_{\sigma(J)} \delta(j, k, l) \theta(m(j, k, l)-f M(j, k, l)) \\
& \times \theta\left(R_{j k}-D^{2}\right) \theta\left(R_{j l}-D^{2}\right) \theta\left(R_{k l}-D^{2}\right) .
\end{aligned}
$$

Notice that this quantity is identical to the $\mathcal{S}_{3}$ function of eq. (2.24), but for the fact that the indices $j, k$ and $l$ can also take the value 6 (and the value $i$ is excluded). Eq. (2.49) has an obvious physical meaning: when a parton gets soft, the remaining partons act as physical jets. The measurement function has then to coincide with the one defined for three partons in the final state. We also point out that each term in the sum in the RHS of eq. (2.47) has a well defined soft limit:

$$
\begin{aligned}
& \lim _{k_{i}^{0} \rightarrow 0} \mathcal{S}_{i}^{(0)}=\mathcal{S}_{3}([i]) \theta\left(R_{i j}-D^{2}\right) \theta\left(R_{i k}-D^{2}\right) \theta\left(R_{i l}-D^{2}\right), \\
& \lim _{k_{i}^{0} \rightarrow 0} \mathcal{S}_{i j}^{(1)}=\mathcal{S}_{3}([i]) \theta\left(D^{2}-R_{i j}\right) \theta\left(R_{i k}-R_{i j}\right) \theta\left(R_{i l}-R_{i j}\right) .
\end{aligned}
$$

- $i \|$ 1. From eqs. (2.39)-(2.42) and eq. (2.44), we have

$$
\lim _{\vec{k}_{i} \| \vec{k}_{1}} \mathcal{S}_{4}=\lim _{\vec{k}_{i} \| \vec{k}_{1}} \mathcal{S}_{i}^{(0)}
$$

It is quite easy to prove that, in this limit, the following equation holds

$$
\lim _{\vec{k}_{i} \| \vec{k}_{1}} \theta(m([i])-i)=1
$$

and therefore

$$
\lim _{\vec{k}_{i} \| \vec{k}_{1}} \mathcal{S}_{4}=\mathcal{S}_{3}([i]) .
$$

The case in which $i \| 2$ is completely analogous and gives an identical result.

- $i \| j$. From eqs. (2.39)-(2.42) and eq. (2.44), we have

$$
\lim _{\vec{k}_{i} \| \vec{k}_{j}} \mathcal{S}_{4}=\lim _{\vec{k}_{i} \| \vec{k}_{j}} \mathcal{S}_{i j}^{(1)}
$$

where we have used the fact that $\mathcal{S}_{i j}^{(1)}$ is symmetric in the exchange of $i$ and $j$ and

$$
\theta\left(d_{i}-d_{j}\right)+\theta\left(d_{j}-d_{i}\right) \equiv 1
$$


In the limit at hand, $d_{i j} \rightarrow 0$ and therefore

$$
\lim _{\vec{k}_{i} \| \vec{k}_{j}} \theta(m([i j])-i j)=1
$$

while all the other $\theta$ functions containing $d_{i j}$ in the list over which the minimum is evaluated are zero. Therefore we have

$$
\lim _{\vec{k}_{i} \| \vec{k}_{j}} \mathcal{S}_{4}=\mathcal{S}_{3}([i j])
$$

where

$$
\begin{aligned}
\mathcal{S}_{3}([i j])= & \sum_{\sigma(J)} \delta(p, k, l) \theta(m(p, k, l)-f M(p, k, l)) \\
& \times \theta\left(R_{p k}-D^{2}\right) \theta\left(R_{p l}-D^{2}\right) \theta\left(R_{k l}-D^{2}\right) .
\end{aligned}
$$

and the indices $p, k$ and $l$ in the sum can now take also the value 7 , having defined $k_{7}=k_{i}+k_{j}$ (notice that this formal manipulation allows to maintain the notation used in the previous case; the meaning of eq.(2.59) is that the final state collinear limit of the $\mathcal{S}_{4}$ function is again a jet-defining function of three partons into three jets, where one of the partons has four-momentum equal to the sum of the four-momenta of the partons becoming collinear).

We finally point out that in the numerical implementation of the algorithm we will also need to consider the case where one parton is soft and collinear to an incoming parton or to a final state parton. In this case, we find

$$
\begin{aligned}
& \lim _{\vec{k}_{i} \| \vec{k}_{1}} \lim _{k_{i}^{0} \rightarrow 0} \mathcal{S}_{i}^{(0)}=\mathcal{S}_{3}([i]), \\
& \lim _{\vec{k}_{i} \| \vec{k}_{j} k_{i} \lim _{i} \rightarrow 0} \mathcal{S}_{i j}^{(1)}=\mathcal{S}_{3}([i j]) .
\end{aligned}
$$

Notice that in eqs. (2.61) and (2.62) the order in which the limits are taken is irrelevant. Also, eq. (2.61) holds true when $i \| 2$.

In the following, we will use the properties of the measurement function $\mathcal{S}_{4}$ to disentangle the structure of the singularities in the real contribution. In spite of this fact, our method is completely general. In fact, we will basically rely only upon eqs. (2.44)-(2.46), that is, on the decomposition of the measurement function into terms which get contribution from two singular regions at the worst. It should be clear that such a decomposition can always be performed, being essentially due to the infrared safeness requirement on the measurement function. 


\subsection{Organization of the calculation}

Given the definitions of the measurement functions and of the collinear counterterms, the main problem we must tackle now is to arrange for all the singularities appearing in the perturbatively calculated cross sections to cancel analytically. Notice that this cancellation is achieved only after that the real and the virtual contributions are summed together, as in eq. (2.14). Also, it must take place already at the partonic level, that is, we do not need to take into account the convolution with the partonic densities in the intermediate steps of the calculation (to have a physical picture of this fact, one can imagine to have very peaked partonic distribution functions, becoming a $\delta(1-x)$ in some limit). A possible choice for the reference frame in which the calculation is carried out is that of the partonic center-of-mass one; this results in a simplification of the kinematics. We begin by considering the singular structure of the cross section; it is apparent from their definition that the measurement functions are boost invariant; therefore, proving the cancellation of the singularities in the partonic center-of-mass frame, ensures that the same cancellation holds true also in the hadronic center-of-mass frame. After the cancellation of singularities, we are left with a subtracted partonic cross section, analogous to $d \hat{\sigma}_{a b}$ of eq. (2.1), defined in the partonic center-of-mass frame instead of the hadronic center-of-mass frame. Clearly, the two are related by a longitudinal boost. The simplest way to perform this boost is by numerical methods (we stress that there is no loss of generality: in all practical cases, the integration in eq. (2.1) has to be carried out numerically).

Since the real and virtual part of the cross section are both divergent, we have to write them in a form in which the structure of the divergencies is very clearly displayed. To this end, the most involved case is that of the real contribution. Taking into account eq. (2.44), we split the real part of the cross section as follows

$$
d \sigma_{a_{1} a_{2}}^{(r)}=\sum_{i}\left(d \sigma_{a_{1} a_{2}, i}^{(s i n g)}+d \sigma_{a_{1} a_{2}, i}^{(f i n)}\right)
$$

where

$$
\begin{aligned}
d \sigma_{a_{1} a_{2}, i}^{(f i n)} & =\frac{1}{4 !} \sum_{\left\{a_{l}\right\}_{3,6}} \mathcal{M}^{(4)}\left(\left\{a_{l}\right\}_{1,6}\right) \mathcal{S}_{i}^{(f i n)} d \phi_{4}, \\
d \sigma_{a_{1} a_{2}, i}^{(s i n g)} & =\frac{1}{4 !} \sum_{\left\{a_{l}\right\}_{3,6}} \mathcal{M}^{(4)}\left(\left\{a_{l}\right\}_{1,6}\right) \mathcal{S}_{i}^{(\text {sing })} d \phi_{4} .
\end{aligned}
$$

$d \sigma_{a_{1} a_{2}, i}^{(f i n)}$ in eq. (2.64) is finite, and it is already suited for numerical computations. On the other hand, all the singular contributions are contained in $d \sigma_{a_{1} a_{2}, i}^{(s i n g)}$, eq. (2.65). 
The form of $\mathcal{S}_{i}^{(s i n g)}$ implies that $d \sigma_{a_{1} a_{2}, i}^{(\text {sing })}$ has the following singularities: $i$ soft, $i \| 1$, $i \| 2$ and $i \| j, \forall j \neq i$. From the previous discussion, we know that the singularities due to initial state collinear emission are cancelled by the addition of the collinear counterterms, eqs. (2.19) and (2.20). It is then useful to define a subtracted real contribution in the following way

$$
d \hat{\sigma}_{a_{1} a_{2}}^{(r)}=d \sigma_{a_{1} a_{2}}^{(r)}+d \sigma_{a_{1} a_{2}}^{(c n t+)}+d \sigma_{a_{1} a_{2}}^{(c n t-)}
$$

that is

$$
d \hat{\sigma}_{a_{1} a_{2}}^{(r)}=\sum_{i}\left(d \hat{\sigma}_{a_{1} a_{2}, i}^{(s i n g)}+d \sigma_{a_{1} a_{2}, i}^{(f i n)}\right)
$$

where

$$
d \hat{\sigma}_{a_{1} a_{2}, i}^{(s i n g)}=d \sigma_{a_{1} a_{2}, i}^{(s i n g)}+\frac{1}{4} d \sigma_{a_{1} a_{2}}^{(c n t+)}+\frac{1}{4} d \sigma_{a_{1} a_{2}}^{(c n t-)} .
$$

The key point here is that no parton but $i$ can be soft. This amounts to a very clean disentangling of the soft regions, and allows to factor out immediately the pure soft singularities, as we will show later.

In the following sections, we will first summarize the results for the virtual contribution, known from the literature. Next, we will discuss the separation of the soft and collinear singularities in the real contribution. We will then be able to analytically evaluate the structure of these singularities. Finally, we will collect the results in a form suitable for numerical evaluation.

\section{Virtual contribution}

Although the calculation of the loop corrections to the two-to-three partonic subprocesses is quite involved, and the result is rather complicated, the structure of the divergent terms is indeed very simple (see e.g. refs. [19,27,28 )

$$
\mathcal{M}^{(3,1)}\left(\left\{a_{l}\right\}_{1,5} ;\left\{k_{l}\right\}_{1,5}\right)=\frac{\alpha_{S}}{2 \pi} \frac{(4 \pi)^{\epsilon}}{\Gamma(1-\epsilon)}\left(\frac{\mu^{2}}{Q^{2}}\right)^{\epsilon} \sum_{\left\{a_{l}\right\}_{3,5}} \mathcal{V}\left(\left\{a_{l}\right\}_{1,5} ;\left\{k_{l}\right\}_{1,5}\right)
$$

where

$$
\begin{aligned}
\mathcal{V}\left(\left\{a_{l}\right\}_{1,5} ;\left\{k_{l}\right\}_{1,5}\right)= & -\left(\frac{1}{\epsilon^{2}} \sum_{n=1}^{5} C\left(a_{n}\right)+\frac{1}{\epsilon} \sum_{n=1}^{5} \gamma\left(a_{n}\right)\right) \mathcal{M}^{(3,0)}\left(\left\{a_{l}\right\}_{1,5} ;\left\{k_{l}\right\}_{1,5}\right) \\
& +\frac{1}{2 \epsilon} \sum_{\substack{n, m=1 \\
n \neq m}}^{5} \log \frac{2 k_{n} \cdot k_{m}}{Q^{2}} \frac{1}{8 \pi^{2}} \mathcal{M}_{m n}^{(3,0)}\left(\left\{a_{l}\right\}_{1,5} ;\left\{k_{l}\right\}_{1,5}\right) \\
& +\mathcal{M}_{N S}^{(3,1)}\left(\left\{a_{l}\right\}_{1,5} ;\left\{k_{l}\right\}_{1,5}\right) .
\end{aligned}
$$


In these equations, $\mu$ is the renormalization scale and $Q$ is an arbitrary mass scale, introduced by Ellis and Sexton in ref. 28] to facilitate the writing of the result. The quantities $\mathcal{M}^{(3,1)}$ and $\mathcal{M}^{(3,0)}$ were defined respectively in eqs. (2.17) and (2.10); the $\mathcal{M}_{m n}^{(3,0)}$ are usually denoted as color-linked Born squared amplitudes. They are symmetric in $m, n$ and satisfy the identity

$$
\sum_{\substack{n=1 \\ n \neq m}}^{5} \mathcal{M}_{m n}^{(3,0)}\left(\left\{a_{l}\right\}_{1,5} ;\left\{k_{l}\right\}_{1,5}\right)=16 C\left(a_{m}\right) \pi^{2} \mathcal{M}^{(3,0)}\left(\left\{a_{l}\right\}_{1,5} ;\left\{k_{l}\right\}_{1,5}\right)
$$

the reason for the $1 / 8 \pi^{2}$ normalization factor (that can be freely chosen, provided that eq. (3.3) is suitably modified) inserted in front of these terms in eq. (3.2) will become clear in the following. The factor

$$
\frac{(4 \pi)^{\epsilon}}{\Gamma(1-\epsilon)}=\frac{\Gamma(1+\epsilon) \Gamma(1-\epsilon)^{2}}{\Gamma(1-2 \epsilon)}+\mathcal{O}\left(\epsilon^{3}\right)
$$

naturally results from loop integration. In eq. (3.2) we also made use of the flavour dependent quantities $C\left(a_{n}\right)$ and $\gamma\left(a_{n}\right)$; for $S U\left(N_{c}\right)$ color group, they are

$$
\begin{array}{ll}
C(g)=C_{A}=N_{c}, & \gamma(g)=\frac{11 C_{A}-4 T_{F} N_{f}}{6} \\
C(q)=C_{F}=\frac{N_{c}^{2}-1}{2 N_{c}}, & \gamma(q)=\frac{3}{2} C_{F}
\end{array}
$$

where $T_{F}=1 / 2$ and $N_{f}$ is the number of quark flavours. All the non-divergent terms in eq. (3.2) were collected in $\mathcal{M}_{N S}^{(3,1)}$. The explicit results can be found in ref. 112 for $5 \mathrm{~g}$ process, ref. 13 for $4 \mathrm{q} 1 \mathrm{~g}$ process, ref. 114,15 for 3g2q process; the calculations were carried out using helicity amplitude methods in the dimensional reduction (DR) scheme. In the DR scheme, $\mathcal{M}^{(3,0)}$ and $\mathcal{M}_{m n}^{(3,0)}$ are evaluated in four dimensions, while in the conventional dimensional regularization scheme (CDR) they are in $4-2 \epsilon$ dimensions. As pointed out in ref. [29], to convert the results obtained in the DR scheme [12 15] into the CDR scheme, one has to use $\mathcal{M}^{(3,0)}$ and $\mathcal{M}_{m n}^{(3,0)}$ in $4-2 \epsilon$ dimensions instead of four dimensions; furthermore, the finite part is modified as follows

$$
\mathcal{M}_{N S}^{(3,1)}(\mathrm{CDR})=\mathcal{M}_{N S}^{(3,1)}(\mathrm{DR})-\mathcal{M}^{(3,0)} \sum_{n=1}^{5} \tilde{\gamma}\left(a_{n}\right),
$$

where the universal factors $\tilde{\gamma}$ are

$$
\tilde{\gamma}(g)=\frac{N_{c}}{6}, \quad \tilde{\gamma}(q)=\frac{1}{2} \frac{N_{c}^{2}-1}{2 N_{c}} .
$$




\section{Real contribution}

\subsection{Separation of singularities}

We now turn to the real contribution, defined in eq. (2.16) and further decomposed in eq. (2.63). Thanks to the fact that no divergencies are present in eq. (2.64), we will deal only with eq. (2.65). In the partonic center-of-mass frame, the incoming partons have momentum

$$
\begin{aligned}
& k_{1}=\frac{\sqrt{S}}{2}(1, \overrightarrow{0}, 1), \\
& k_{2}=\frac{\sqrt{S}}{2}(1, \overrightarrow{0},-1),
\end{aligned}
$$

where $\sqrt{S}$ is the partonic center-of-mass energy and $\overrightarrow{0}$ is the null vector in a $(2-2 \epsilon)$ dimensional space. In this frame, we write the momentum of parton $i$ as

$$
k_{i}=\frac{\sqrt{S}}{2} \xi_{i}\left(1, \sqrt{1-y_{i}^{2}} \vec{e}_{i T}, y_{i}\right)
$$

where $\vec{e}_{i T}$ is a unit vector in the $(2-2 \epsilon)$-dimensional transverse momentum space, $-1 \leq y_{i} \leq 1$ and $0 \leq \xi_{i} \leq 1$. By construction, when $\xi_{i} \rightarrow 0$ the parton $i$ gets soft, and when $y_{i} \rightarrow \pm 1$ it gets collinear to the incoming partons. With this parametrization, in $4-2 \epsilon$ dimensions the invariant measure over the variables of parton $i$ is

$$
d \phi(i)=\frac{d^{3-2 \epsilon} k_{i}}{(2 \pi)^{3-2 \epsilon} 2 k_{i}^{0}}=\frac{1}{2(2 \pi)^{3-2 \epsilon}}\left(\frac{\sqrt{S}}{2}\right)^{2-2 \epsilon} \xi_{i}^{1-2 \epsilon}\left(1-y_{i}^{2}\right)^{-\epsilon} d \xi_{i} d y_{i} d \Omega_{i}^{(2-2 \epsilon)}
$$

where $d \Omega_{i}^{(2-2 \epsilon)}$ is the angular measure in $2-2 \epsilon$ dimensions. We also write the fourbody phase space as $d \phi_{4}=d \phi d \phi(i)$, where

$$
d \phi=(2 \pi)^{4-2 \epsilon} \delta^{4-2 \epsilon}\left(k_{1}+k_{2}-\sum_{l=3}^{6} k_{l}\right) \prod_{l}^{[i]} \frac{d^{3-2 \epsilon} k_{l}}{(2 \pi)^{3-2 \epsilon} 2 k_{l}^{0}} .
$$

We now regulate the soft singularities by multiplying the invariant amplitude squared by $\xi_{i}^{2}$. Taking into account eq. (4.4), eq. (2.65) becomes

$$
\begin{aligned}
d \sigma_{a_{1} a_{2}, i}^{(s i n g)} & =\frac{\xi_{i}^{2}}{4 !} \sum_{\left\{a_{l}\right\}_{3,6}} \mathcal{M}^{(4)}\left(\left\{a_{l}\right\}_{1,6}\right) \mathcal{S}_{i}^{(\text {sing })} d \phi \\
& \times \frac{1}{2(2 \pi)^{3-2 \epsilon}}\left(\frac{\sqrt{S}}{2}\right)^{2-2 \epsilon} \xi_{i}^{-1-2 \epsilon}\left(1-y_{i}^{2}\right)^{-\epsilon} d \xi_{i} d y_{i} d \Omega_{i}^{(2-2 \epsilon)}
\end{aligned}
$$


We can make use of the following identity

$$
\xi_{i}^{-1-2 \epsilon}=-\frac{\xi_{c u t}^{-2 \epsilon}}{2 \epsilon} \delta\left(\xi_{i}\right)+\left(\frac{1}{\xi_{i}}\right)_{c}-2 \epsilon\left(\frac{\log \xi_{i}}{\xi_{i}}\right)_{c}+\mathcal{O}\left(\epsilon^{2}\right),
$$

where $\xi_{\text {cut }}$ is an arbitrary parameter satisfying the condition $0<\xi_{\text {cut }} \leq 1$, and the distributions in the RHS are defined as follows

$$
\begin{gathered}
<\left(\frac{1}{\xi_{i}}\right)_{c}, f>=\int_{0}^{1} d \xi_{i} \frac{f\left(\xi_{i}\right)-f(0) \theta\left(\xi_{c u t}-\xi_{i}\right)}{\xi_{i}} \\
<\left(\frac{\log \xi_{i}}{\xi_{i}}\right)_{c}, f>=\int_{0}^{1} d \xi_{i}\left[f\left(\xi_{i}\right)-f(0) \theta\left(\xi_{c u t}-\xi_{i}\right)\right] \frac{\log \xi_{i}}{\xi_{i}} .
\end{gathered}
$$

Notice that different $\xi_{\text {cut }}$ can be chosen for different $i$; here we restricted to the simplest case. Substituting eq. (4.7) into eq. (4.6) we get

$$
d \sigma_{a_{1} a_{2}, i}^{(s i n g)}=d \sigma_{a_{1} a_{2}, i}^{(s)}+d \sigma_{a_{1} a_{2}, i}^{(n s)}
$$

where

$$
\begin{aligned}
d \sigma_{a_{1} a_{2}, i}^{(s)} & =-\frac{\xi_{c u t}^{-2 \epsilon}}{2 \epsilon} \delta\left(\xi_{i}\right) d \xi_{i}\left(\frac{\xi_{i}^{2}}{4 !} \sum_{\left\{a_{l}\right\}_{3,6}} \mathcal{M}^{(4)}\left(\left\{a_{l}\right\}_{1,6}\right)\right) \mathcal{S}_{i}^{(\text {sing })} d \phi \\
& \times \frac{1}{2(2 \pi)^{3-2 \epsilon}}\left(\frac{\sqrt{S}}{2}\right)^{2-2 \epsilon}\left(1-y_{i}^{2}\right)^{-\epsilon} d y_{i} d \Omega_{i}^{(2-2 \epsilon)}, \\
d \sigma_{a_{1} a_{2}, i}^{(n s)} & =\left[\left(\frac{1}{\xi_{i}}\right)_{c}-2 \epsilon\left(\frac{\log \xi_{i}}{\xi_{i}}\right)_{c}\right]\left(\frac{\xi_{i}^{2}}{4 !} \sum_{\left\{a_{l}\right\}_{3,6}} \mathcal{M}^{(4)}\left(\left\{a_{l}\right\}_{1,6}\right)\right) \mathcal{S}_{i}^{(s i n g)} d \phi \\
& \times \frac{1}{2(2 \pi)^{3-2 \epsilon}}\left(\frac{\sqrt{S}}{2}\right)^{2-2 \epsilon}\left(1-y_{i}^{2}\right)^{-\epsilon} d \xi_{i} d y_{i} d \Omega_{i}^{(2-2 \epsilon)} .
\end{aligned}
$$

Eq. (4.11) does contain the singularities due to parton $i$ becoming soft, while eq. 4.12) is free of them, but does contain collinear singularities. We can also fully disentangle the collinear singularities of $d \sigma_{a_{1} a_{2}, i}^{(n s)}$. Using eq. (2.39) and eq. 2.40) we notice that a single term in the $\mathcal{S}_{i}^{(s i n g)}$ function gets contributions from one singular collinear region at the worst. Therefore, using eq. (2.45), we can naturally split $d \sigma_{a_{1} a_{2}, i}^{(n s)}$ into several terms:

$$
d \sigma_{a_{1} a_{2}, i}^{(n s)}=d \sigma_{a_{1} a_{2}, i}^{(i n)}+\sum_{j}^{[i]} d \sigma_{a_{1} a_{2}, i j}^{(o u t)}
$$


where

$$
\begin{aligned}
d \sigma_{a_{1} a_{2}, i}^{(i n)} & =\left[\left(\frac{1}{\xi_{i}}\right)_{c}-2 \epsilon\left(\frac{\log \xi_{i}}{\xi_{i}}\right)_{c}\right]\left(\frac{\xi_{i}^{2}}{4 !} \sum_{\left\{a_{l}\right\}_{3,6}} \mathcal{M}^{(4)}\left(\left\{a_{l}\right\}_{1,6}\right)\right) \mathcal{S}_{i}^{(0)} d \phi \\
& \times \frac{1}{2(2 \pi)^{3-2 \epsilon}}\left(\frac{\sqrt{S}}{2}\right)^{2-2 \epsilon}\left(1-y_{i}^{2}\right)^{-\epsilon} d \xi_{i} d y_{i} d \Omega_{i}^{(2-2 \epsilon)}, \\
d \sigma_{a_{1} a_{2}, i j}^{(\text {out })} & =\left[\left(\frac{1}{\xi_{i}}\right)_{c}-2 \epsilon\left(\frac{\log \xi_{i}}{\xi_{i}}\right)_{c}\right]\left(\frac{\xi_{i}^{2}}{4 !} \sum_{\left\{a_{l}\right\}_{3,6}} \mathcal{M}^{(4)}\left(\left\{a_{l}\right\}_{1,6}\right)\right) \mathcal{S}_{i j}^{(1)} \theta\left(d_{j}-d_{i}\right) d \phi \\
& \times \frac{1}{2(2 \pi)^{3-2 \epsilon}}\left(\frac{\sqrt{S}}{2}\right)^{2-2 \epsilon}\left(1-y_{i}^{2}\right)^{-\epsilon} d \xi_{i} d y_{i} d \Omega_{i}^{(2-2 \epsilon)} .
\end{aligned}
$$

By construction, eq. (4.14) contains only initial state collinear singularities, while eq. (4.15) contains only final state ones.

In summary, the decompositions defined in eqs. (2.63), (4.10) and (4.13) allow to write the real contribution to the partonic cross section in the following way

$$
d \sigma_{a_{1} a_{2}}^{(r)}=\sum_{i}\left(d \sigma_{a_{1} a_{2}, i}^{(f i n)}+d \sigma_{a_{1} a_{2}, i}^{(s)}+d \sigma_{a_{1} a_{2}, i}^{(i n)}+\sum_{j}^{[i]} d \sigma_{a_{1} a_{2}, i j}^{(o u t)}\right)
$$

where the quantities in the RHS of this equation have been presented in eqs. (2.64), (4.11), (4.14), and (4.15) respectively. As far as the real contribution is concerned, eq. (4.16) will be regarded as our master equation. The key point is that every term in the RHS gets contributions from two singular regions at the worst. In the next three subsections, we will calculate the singular contributions explicitly. At the very end, we will show that, as expected, the sum of the real contribution, of the virtual contribution and of the collinear counterterms is finite.

\section{2. $\quad$ Soft singularities}

We begin by considering eq. (4.11). The $\delta\left(\xi_{i}\right)$ allows to take the soft limit of all the quantities appearing in that equation. In particular, we have

$$
\lim _{\xi_{i} \rightarrow 0} \mathcal{M}^{(4)}\left(\left\{a_{l}\right\}_{1,6} ;\left\{k_{l}\right\}_{1,6}\right)=\delta_{g a_{i}} \frac{\alpha_{S} \mu^{2 \epsilon}}{2 \pi} \sum_{\substack{n, m \\ n<m}}^{[i]} \frac{k_{n} \cdot k_{m}}{k_{n} \cdot k_{i} k_{m} \cdot k_{i}} \mathcal{M}_{m n}^{(3,0)}\left(\left\{a_{l}\right\}_{1,6}^{[i]} ;\left\{k_{l}\right\}_{1,6}^{[i]}\right)
$$


where $n$ and $m$ in the sum run also over the values 1 and 2 (incoming partons). In eq. (4.17) we inserted $\mu^{2 \epsilon}$ as customary in $4-2 \epsilon$ dimensions. Eq. (4.17) also explains the reason for the normalization of the $\mathcal{M}_{m n}^{(3,0)}$ terms in eq. (3.2); we choose to keep the form of the soft limit as simple as possible. Taking into account eq. (2.49) and the formal limit

$$
\lim _{\xi_{i} \rightarrow 0} d \phi=d \phi_{3}\left(k_{1}, k_{2} \rightarrow\left\{k_{l}\right\}_{3,6}^{[i]}\right)
$$

and using $\sum_{\left\{a_{l}\right\}_{3,6}} \delta_{g a_{i}}=\sum_{\left\{a_{l}\right\}_{3,6}^{[i]}}$, eq. (4.11) becomes therefore

$$
\begin{aligned}
d \sigma_{a_{1} a_{2}, i}^{(s)} & =-\frac{\alpha_{S}}{2 \pi} \frac{\xi_{c u t}^{-2 \epsilon}}{2 \epsilon} \frac{2^{2 \epsilon}}{2(2 \pi)^{3-2 \epsilon}}\left(\frac{S}{\mu^{2}}\right)^{-\epsilon} \\
& \times \sum_{\substack{n, m \\
n<m}}^{[i]} \frac{1}{4 !} \sum_{\substack{\left\{a_{l}\right\}_{3,6}^{[i]} \\
\mathcal{M}_{m n}^{(3,0)}}}\left(\left\{a_{l}\right\}_{1,6}^{[i]} ;\left\{k_{l}\right\}_{1,6}^{[i]}\right) \mathcal{S}_{3}([i]) d \phi_{3}\left(k_{1}, k_{2} \rightarrow\left\{k_{l}\right\}_{3,6}^{[i]}\right) \\
& \times \delta\left(\xi_{i}\right)\left(\frac{\sqrt{S}}{2}\right)^{2} \frac{k_{n} \cdot k_{m}}{k_{n} \cdot k_{i} k_{m} \cdot k_{i}} \xi_{i}^{2}\left(1-y_{i}^{2}\right)^{-\epsilon} d \xi_{i} d y_{i} d \Omega_{i}^{(2-2 \epsilon)} .
\end{aligned}
$$

The dependence of the RHS of eq. (4.19) upon the variables $y_{i}$ and $\Omega_{i}$ is fully contained in the last line. The integral over $d \xi_{i}, d y_{i}$ and $d \Omega_{i}^{(2-2 \epsilon)}$ can therefore be performed explicitly; the result is reported in appendix A. We can cast eq. (4.19) in the following form

$$
\begin{aligned}
d \sigma_{a_{1} a_{2}, i}^{(s)} & =\frac{\alpha_{S}}{2 \pi} \sum_{\substack{n, m \\
n<m}}^{[i]}\left(\mathcal{I}_{m n}^{(d i v)}+\mathcal{I}_{m n}^{(r e g)}\right) \\
& \times \frac{1}{4 !} \sum_{\substack{\left\{a_{l}\right\}_{3,6}^{[i]} \\
\text { (reg }}} \mathcal{M}_{m n}^{(3,0)}\left(\left\{a_{l}\right\}_{1,6}^{[i]} ;\left\{k_{l}\right\}_{1,6}^{[i]}\right) \mathcal{S}_{3}([i]) d \phi_{3}\left(k_{1}, k_{2} \rightarrow\left\{k_{l}\right\}_{3,6}^{[i]}\right)
\end{aligned}
$$

with

$$
\mathcal{I}_{\text {mn }}^{(\text {div })}+\mathcal{I}_{m n}^{(\text {reg })}=-\frac{\xi_{\text {cut }}^{-2 \epsilon}}{2 \epsilon} \frac{2^{2 \epsilon}}{2(2 \pi)^{3-2 \epsilon}}\left(\frac{S}{\mu^{2}}\right)^{-\epsilon} \mathcal{J}_{m n},
$$

where $\mathcal{J}_{m n}$ is given in eq. (A.8). Explicitly

$$
\begin{aligned}
\mathcal{I}_{m n}^{(\text {div })=} \frac{1}{8 \pi^{2}} & \frac{(4 \pi)^{\epsilon}}{\Gamma(1-\epsilon)}\left(\frac{\mu^{2}}{Q^{2}}\right)^{\epsilon}\left[\frac{1}{\epsilon^{2}}-\frac{1}{\epsilon}\left(\log \frac{2 k_{n} \cdot k_{m}}{Q^{2}}-\log \frac{4 E_{n} E_{m}}{\xi_{c u t}^{2} S}\right)\right], \\
\mathcal{I}_{m n}^{(r e g)}=\frac{1}{8 \pi^{2}} & {\left[\frac{1}{2} \log ^{2} \frac{\xi_{\text {cut }}^{2} S}{Q^{2}}+\log \frac{\xi_{\text {cut }}^{2} S}{Q^{2}} \log \frac{k_{n} \cdot k_{m}}{2 E_{n} E_{m}}-\operatorname{Li}_{2}\left(\frac{k_{n} \cdot k_{m}}{2 E_{n} E_{m}}\right)\right.} \\
& \left.+\frac{1}{2} \log ^{2} \frac{2 k_{n} \cdot k_{m}}{E_{n} E_{m}}-\log \left(4-\frac{2 k_{n} \cdot k_{m}}{E_{n} E_{m}}\right) \log \frac{k_{n} \cdot k_{m}}{2 E_{n} E_{m}}-2 \log ^{2} 2\right] .
\end{aligned}
$$


Here $E_{n}$ is the energy of the parton $n$ in the partonic center-of-mass frame and $Q$ is the mass scale introduced in eq. (3.2) . We can express $E_{n}$ in term of invariant quantities through the equation

$$
E_{n}=\frac{k_{1} \cdot k_{n}+k_{2} \cdot k_{n}}{\sqrt{S}} .
$$

It is apparent that the dependence upon the index $i$ in eq. (4.20) is immaterial. We can therefore relabel the partons at will and get

$$
d \sigma_{a_{1} a_{2}}^{(s)}=\sum_{i} d \sigma_{a_{1} a_{2}, i}^{(s)}=\frac{\alpha_{S}}{2 \pi} \frac{1}{2} \sum_{\substack{n, m=1 \\ n \neq m}}^{5}\left(\mathcal{I}_{m n}^{(d i v)}+\mathcal{I}_{m n}^{(r e g)}\right) \sum_{\left\{a_{l}\right\}_{3,5}} d \sigma_{m n}^{(0)}\left(\left\{a_{l}\right\}_{1,5}\right)
$$

where we have set

$$
d \sigma_{m n}^{(0)}\left(\left\{a_{l}\right\}_{1,5} ;\left\{k_{l}\right\}_{1,5}\right)=\frac{1}{3 !} \mathcal{M}_{m n}^{(3,0)}\left(\left\{a_{l}\right\}_{1,5} ;\left\{k_{l}\right\}_{1,5}\right) \mathcal{S}_{3} d \phi_{3}\left(k_{1}, k_{2} \rightarrow\left\{k_{l}\right\}_{3,5}\right)
$$

(in the following, as in eq. (4.25), we will not indicate explicitly the dependence upon the momenta of this quantity). Exploiting eq. (3.3) and using the fact that $E_{1}=E_{2}=\sqrt{S} / 2$, eq. (4.25) becomes

$$
\begin{aligned}
d \sigma_{a_{1} a_{2}}^{(s)}= & \frac{\alpha_{S}}{2 \pi} \frac{(4 \pi)^{\epsilon}}{\Gamma(1-\epsilon)}\left(\frac{\mu^{2}}{Q^{2}}\right)^{\epsilon} \sum_{\left\{a_{l}\right\}_{3,5}}\left[\frac{1}{\epsilon^{2}} \sum_{n=1}^{5} C\left(a_{n}\right)+\frac{2}{\epsilon} \sum_{j=3}^{5} C\left(a_{j}\right) \log \frac{2 E_{j}}{\xi_{c u t} \sqrt{S}}\right. \\
& \left.-\frac{2}{\epsilon}\left(C\left(a_{1}\right)+C\left(a_{2}\right)\right) \log \xi_{c u t}\right] d \sigma^{(0)}\left(\left\{a_{l}\right\}_{1,5}\right) \\
- & \frac{\alpha_{S}}{2 \pi} \frac{(4 \pi)^{\epsilon}}{\Gamma(1-\epsilon)}\left(\frac{\mu^{2}}{Q^{2}}\right)^{\epsilon} \frac{1}{2 \epsilon} \sum_{\substack{n, m=1 \\
n \neq m}}^{5} \log \frac{2 k_{n} \cdot k_{m}}{Q^{2}} \frac{1}{8 \pi^{2}} \sum_{\left\{a_{l}\right\}_{3,5}} d \sigma_{m n}^{(0)}\left(\left\{a_{l}\right\}_{1,5}\right) \\
+ & \frac{\alpha_{S}}{2 \pi} \frac{1}{2} \sum_{\substack{n, m=1 \\
n \neq m}}^{5} \mathcal{I}_{m n}^{(r e g)} \sum_{\left\{a_{l}\right\}_{3,5}} d \sigma_{m n}^{(0)}\left(\left\{a_{l}\right\}_{1,5}\right) .
\end{aligned}
$$

where, consistently with eq. (4.26),

$$
d \sigma^{(0)}\left(\left\{a_{l}\right\}_{1,5} ;\left\{k_{l}\right\}_{1,5}\right)=\frac{1}{3 !} \mathcal{M}^{(3,0)}\left(\left\{a_{l}\right\}_{1,5} ;\left\{k_{l}\right\}_{1,5}\right) \mathcal{S}_{3} d \phi_{3}\left(k_{1}, k_{2} \rightarrow\left\{k_{l}\right\}_{3,5}\right) ;
$$

this quantity is just the Born cross section of eq. (2.6) without the sum over the flavours of the final state partons. We point out that the second and the third singular term in eq. (4.27) are cancelled by similar terms appearing in the final and initial state contributions. The remaining singular terms are cancelled by the virtual contribution. 


\subsection{Initial state singularities}

We now turn to eq. (4.14). The divergencies due to parton $i$ becoming collinear to one of the incoming partons can simultaneously be regulated by multiplying the invariant amplitude squared by the factor $\left(1-y_{i}^{2}\right)$. Eq. (4.14) becomes

$$
\begin{aligned}
d \sigma_{a_{1} a_{2}, i}^{(i n)} & =\frac{1}{4 !}\left[\left(\frac{1}{\xi_{i}}\right)_{c}-2 \epsilon\left(\frac{\log \xi_{i}}{\xi_{i}}\right)_{c}\right]\left(\left(1-y_{i}^{2}\right) \xi_{i}^{2} \sum_{\left\{a_{l}\right\}_{3,6}} \mathcal{M}^{(4)}\left(\left\{a_{l}\right\}_{1,6}\right)\right) \mathcal{S}_{i}^{(0)} d \phi \\
& \times \frac{1}{2(2 \pi)^{3-2 \epsilon}}\left(\frac{\sqrt{S}}{2}\right)^{2-2 \epsilon}\left(1-y_{i}^{2}\right)^{-1-\epsilon} d \xi_{i} d y_{i} d \Omega_{i}^{(2-2 \epsilon)}
\end{aligned}
$$

We use the identity

$$
\left(1-y_{i}^{2}\right)^{-1-\epsilon}=-\frac{\left(2 \delta_{I}\right)^{-\epsilon}}{2 \epsilon}\left[\delta\left(1-y_{i}\right)+\delta\left(1+y_{i}\right)\right]+\mathcal{P}\left(y_{i}\right)+\mathcal{O}(\epsilon)
$$

where

$$
\mathcal{P}\left(y_{i}\right)=\frac{1}{2}\left[\left(\frac{1}{1-y_{i}}\right)_{\delta_{I}}+\left(\frac{1}{1+y_{i}}\right)_{\delta_{I}}\right] .
$$

Here $\delta_{I}$ is an arbitrary parameter satisfying the condition $0<\delta_{I} \leq 2$, and we defined

$$
\begin{aligned}
& <\left(\frac{1}{1-y_{i}}\right)_{\delta_{I}}, f>=\int_{-1}^{1} d y_{i} \frac{f\left(y_{i}\right)-f(1) \theta\left(y_{i}-1+\delta_{I}\right)}{1-y_{i}} \\
& <\left(\frac{1}{1+y_{i}}\right)_{\delta_{I}}, f>=\int_{-1}^{1} d y_{i} \frac{f\left(y_{i}\right)-f(-1) \theta\left(-y_{i}-1+\delta_{I}\right)}{1+y_{i}} .
\end{aligned}
$$

Very much like the $\xi_{\text {cut }}$ parameter, it would be possible to choose a different $\delta_{I}$ for every $i$; we prefer however to avoid the proliferation of such parameters. Eq. (4.29) can be splitted into three terms, the first two which contain the collinear singularities (and in which the invariant amplitude is substituted with its collinear limit), and the third one which is finite in the limits $y_{i} \rightarrow \pm 1$. Explicitly

$$
d \sigma_{a_{1} a_{2}, i}^{(i n)}=d \sigma_{a_{1} a_{2}, i}^{(i n,+)}+d \sigma_{a_{1} a_{2}, i}^{(i n,-)}+d \sigma_{a_{1} a_{2}, i}^{(i n, f)}
$$

where

$$
\begin{aligned}
d \sigma_{a_{1} a_{2}, i}^{(i n, f)} & =\frac{1}{4 !} \mathcal{P}\left(y_{i}\right)\left[\left(\frac{1}{\xi_{i}}\right)_{c}-2 \epsilon\left(\frac{\log \xi_{i}}{\xi_{i}}\right)_{c}\right] \frac{1}{2(2 \pi)^{3-2 \epsilon}}\left(\frac{\sqrt{S}}{2}\right)^{2-2 \epsilon} \\
& \times\left(\left(1-y_{i}^{2}\right) \xi_{i}^{2} \sum_{\left\{a_{l}\right\}_{3,6}} \mathcal{M}^{(4)}\left(\left\{a_{l}\right\}_{1,6}\right)\right) \mathcal{S}_{i}^{(0)} d \phi d \xi_{i} d y_{i} d \Omega_{i}^{(2-2 \epsilon)}
\end{aligned}
$$


and

$$
\begin{aligned}
d \sigma_{a_{1} a_{2}, i}^{(i n, \pm)} & =-\frac{\left(2 \delta_{I}\right)^{-\epsilon}}{2 \epsilon} \delta\left(1 \mp y_{i}\right) \frac{1}{4 !}\left[\left(\frac{1}{\xi_{i}}\right)_{c}-2 \epsilon\left(\frac{\log \xi_{i}}{\xi_{i}}\right)_{c}\right] \frac{1}{2(2 \pi)^{3-2 \epsilon}}\left(\frac{\sqrt{S}}{2}\right)^{2-2 \epsilon} \\
& \times\left(\left(1-y_{i}^{2}\right) \xi_{i}^{2} \sum_{\left\{a_{l}\right\}_{3,6}} \mathcal{M}^{(4)}\left(\left\{a_{l}\right\}_{1,6}\right)\right) \mathcal{S}_{i}^{(0)} d \phi d \xi_{i} d y_{i} d \Omega_{i}^{(2-2 \epsilon)}
\end{aligned}
$$

Thanks to the subtraction prescriptions of $\mathcal{P}\left(y_{i}\right)$, which regulate initial state divergencies, and to eq. (2.39), which guarantees that $\mathcal{S}_{i}^{(0)}$ does not get contributions from final state collinear divergencies, eq. (4.35) is finite, and therefore we can set $\epsilon=0$. We get

$$
\begin{aligned}
d \sigma_{a_{1} a_{2}, i}^{(i n, f)} & =\frac{1}{4 !} \frac{1}{2}\left(\frac{1}{\xi_{i}}\right)_{c}\left[\left(\frac{1}{1-y_{i}}\right)_{\delta_{I}}+\left(\frac{1}{1+y_{i}}\right)_{\delta_{I}}\right] \frac{1}{2(2 \pi)^{3}}\left(\frac{\sqrt{S}}{2}\right)^{2} \\
& \times\left(\left(1-y_{i}^{2}\right) \xi_{i}^{2} \sum_{\left\{a_{l}\right\}_{3,6}} \mathcal{M}^{(4)}\left(\left\{a_{l}\right\}_{1,6}\right)\right) \mathcal{S}_{i}^{(0)} d \phi d \xi_{i} d y_{i} d \varphi_{i} .
\end{aligned}
$$

Since all the divergencies have been properly regulated, the RHS of eq. (4.37) can be numerically integrated.

We still have to deal with the divergent part of eq. (4.34), that is to say, with the quantities defined in eq. (4.36). The $\delta\left(1 \mp y_{i}\right)$ in eq. (4.36) allows to take the appropriate collinear limit of all the quantities appearing in that equation. We only consider $d \sigma_{a_{1} a_{2}, i}^{(i n,+)}$, that is the case in which $i \| 1$; the treatment of $i \| 2$ is completely analogous. The collinear limit of the invariant amplitude squared can be written as (see appendix B)

$$
\begin{aligned}
\lim _{y_{i} \rightarrow 1} \mathcal{M}^{(4)}\left(\left\{a_{l}\right\}_{1,6} ;\left\{k_{l}\right\}_{1,6}\right)=\frac{4 \pi \alpha_{S} \mu^{2 \epsilon}}{k_{i} \cdot k_{1}} \Delta\left(\left\{a_{l}\right\}_{1,6} ;\left\{k_{l}\right\}_{1,6}\right) \\
\quad+\frac{4 \pi \alpha_{S} \mu^{2 \epsilon}}{k_{i} \cdot k_{1}} P_{S\left(a_{1}, \bar{a}_{i}\right) a_{1}}^{<}(z, \epsilon) \mathcal{M}^{(3,0)}\left(S\left(a_{1}, \bar{a}_{i}\right), a_{2},\left\{a_{l}\right\}_{3,6}^{[i]} ; z k_{1}, k_{2},\left\{k_{l}\right\}_{3,6}^{[i]}\right),
\end{aligned}
$$

where $P_{a b}^{<}(z, \epsilon)$ is the Altarelli-Parisi kernel for $z<1$ in $4-2 \epsilon$ dimensions and $S(c, d)$ is the flavour of the parton which can split into two partons of flavour $c$ and $d$; for example, $S(g, g)=g$ and $S(q, g)=q$; by definition, if the splitting into the flavours $c$ and $d$ is not possible, $P_{S(c, d) a}^{<}$is zero. The $z$ parameter is such that, in the collinear limit, $k_{i}=(1-z) k_{1}$. Taking into account eq. (4.3) we have

$$
\xi_{i} \equiv 1-z \quad \text { if } \quad y_{i}=1
$$


Using eq. (4.38), we can then write

$$
\begin{aligned}
\lim _{y_{i} \rightarrow 1}\left(1-y_{i}^{2}\right) & \xi_{i}^{2} \mathcal{M}^{(4)}\left(\left\{a_{l}\right\}_{1,6} ;\left\{k_{l}\right\}_{1,6}\right)=8 \pi \alpha_{S} \mu^{2 \epsilon}\left(\frac{2}{\sqrt{S}}\right)^{2} \xi_{i} \Delta\left(\left\{a_{l}\right\}_{1,6} ;\left\{k_{l}\right\}_{1,6}\right) \\
& +8 \pi \alpha_{S} \mu^{2 \epsilon}\left(\frac{2}{\sqrt{S}}\right)^{2} \xi_{i} P_{S\left(a_{1}, \bar{a}_{i}\right) a_{1}}^{<}\left(1-\xi_{i}, \epsilon\right) \\
& \times \mathcal{M}^{(3,0)}\left(S\left(a_{1}, \bar{a}_{i}\right), a_{2},\left\{a_{l}\right\}_{3,6}^{[i]} ;\left(1-\xi_{i}\right) k_{1}, k_{2},\left\{k_{l}\right\}_{3,6}^{[i]}\right)
\end{aligned}
$$

In this equation, the dependence of $\Delta$ upon the azimuthal variables of parton $i$ is trivial; therefore, it can be shown that

$$
\int d \Omega_{i}^{(2-2 \epsilon)} \Delta=0
$$

Furthermore, the second term in eq. (4.40) does not depend upon the azimuthal variables, and after the $d \Omega_{i}^{(2-2 \epsilon)}$ integration in eq. (4.36) we get a factor

$$
\int d \Omega_{i}^{(2-2 \epsilon)}=\frac{2 \pi^{1-\epsilon}}{\Gamma(1-\epsilon)}
$$

Putting all the numerical factors together, taking into account the formal limit

$$
\lim _{\vec{k}_{i} \| \vec{k}_{1}} d \phi=d \phi_{3}\left(\left(1-\xi_{i}\right) k_{1}, k_{2} \rightarrow\left\{k_{l}\right\}_{3,6}^{[i]}\right)
$$

using eq. (2.55) and

$$
\frac{1}{\bar{\epsilon}}=\frac{1}{\epsilon}-\gamma_{E}+\log 4 \pi
$$

we get

$$
\begin{aligned}
d \sigma_{a_{1} a_{2}, i}^{(i n,+)} & =-\frac{\alpha_{S}}{2 \pi} \sum_{\left\{a_{l}\right\}_{3,6}}\left(\frac{1}{\bar{\epsilon}}-\log \frac{S \delta_{I}}{2 \mu^{2}}\right)\left[\left(\frac{1}{\xi_{i}}\right)_{c}-2 \epsilon\left(\frac{\log \xi_{i}}{\xi_{i}}\right)_{c}\right]_{i} \xi_{S\left(a_{1}, \bar{a}_{i}\right) a_{1}}^{<}\left(1-\xi_{i}, \epsilon\right) \\
& \times \frac{1}{4 !} \mathcal{M}^{(3,0)}\left(S\left(a_{1}, \bar{a}_{i}\right), a_{2},\left\{a_{l}\right\}_{3,6}^{[i]} ;\left(1-\xi_{i}\right) k_{1}, k_{2},\left\{k_{l}\right\}_{3,6}^{[i]}\right) \\
& \times \mathcal{S}_{3}([i]) d \phi_{3}\left(\left(1-\xi_{i}\right) k_{1}, k_{2} \rightarrow\left\{k_{l}\right\}_{3,6}^{[i]}\right) d \xi_{i} .
\end{aligned}
$$

When the sum over $a_{i}$ is performed in eq. (4.45), the quantity $S\left(a_{1}, \bar{a}_{i}\right)$ takes all the possible flavour values such that $P_{S\left(a_{1}, \bar{a}_{i}\right) a_{1}}^{<}$is different from zero. Therefore, eq. (4.45) 
can be rewritten as

$$
\begin{aligned}
d \sigma_{a_{1} a_{2}, i}^{(i n,+)} & =-\frac{\alpha_{S}}{2 \pi} \sum_{d}\left(\frac{1}{\bar{\epsilon}}-\log \frac{S \delta_{I}}{2 \mu^{2}}\right)\left[\left(\frac{1}{\xi_{i}}\right)_{c}-2 \epsilon\left(\frac{\log \xi_{i}}{\xi_{i}}\right)_{c}\right]_{i} \xi_{i} P_{d a_{1}}^{<}\left(1-\xi_{i}, \epsilon\right) \\
& \times \frac{1}{4 !} \sum_{\left\{a_{l}\right\}_{3,6}^{[i]}} \mathcal{M}^{(3,0)}\left(d, a_{2},\left\{a_{l}\right\}_{3,6}^{[i]} ;\left(1-\xi_{i}\right) k_{1}, k_{2},\left\{k_{l}\right\}_{3,6}^{[i]}\right) \\
& \times \mathcal{S}_{3}([i]) d \phi_{3}\left(\left(1-\xi_{i}\right) k_{1}, k_{2} \rightarrow\left\{k_{l}\right\}_{3,6}^{[i]}\right) d \xi_{i} .
\end{aligned}
$$

We can now show that the divergent part of eq. (4.46) is cancelled by the collinear counterterm of eq. (2.19) up to a factor $1 / 4$ (see eq. (2.68) to understand the $1 / 4$ factor). To this purpose, we rewrite eq. (2.19) in terms of the variable $\xi_{i}$. Using eq. (4.39), it is a simple matter of algebra to prove the following identity

$$
\delta\left(1-y_{i}\right)\left(\frac{1}{1-z}\right)_{+}=\delta\left(1-y_{i}\right)\left[\left(\frac{1}{\xi_{i}}\right)_{c}+\delta\left(\xi_{i}\right) \log \xi_{c u t}\right],
$$

where the $\delta\left(1-y_{i}\right)$ formally states the fact that the above equation is strictly valid only in the collinear limit. The Altarelli-Parisi kernel can be put in the form

$$
\begin{aligned}
P_{a b}(z, 0) & =\frac{(1-z) P_{a b}^{<}(z, 0)}{(1-z)_{+}}+\gamma(a) \delta_{a b} \delta(1-z), \\
2 C(a) \delta_{a b} \delta(1-z) & =\delta(1-z)(1-z) P_{a b}^{<}(z, 0),
\end{aligned}
$$

where the quantities $C(a)$ and $\gamma(a)$ were given in eq. (3.5) for gluons and eq. (3.6) for quarks. Using eq. (4.47), (4.48) and (4.49), and changing the integration variable $z \rightarrow 1-\xi_{i}$, it is straightforward to see that eq. (2.19) can be cast in the form

$$
\begin{aligned}
d \sigma_{a_{1} a_{2}}^{(c n t+)}= & \frac{\alpha_{S}}{2 \pi} \sum_{d}\left\{-K_{d a_{1}}\left(1-\xi_{i}\right)\right. \\
& \left.+\frac{1}{\bar{\epsilon}}\left[\left(\frac{1}{\xi_{i}}\right)_{c} \xi_{i} P_{d a_{1}}^{<}\left(1-\xi_{i}, 0\right)+\delta_{d a_{1}} \delta\left(\xi_{i}\right)\left(\gamma(d)+2 C(d) \log \xi_{c u t}\right)\right]\right\} \\
& \times \frac{1}{3 !} \sum_{\left\{a_{l}\right\}_{3,6}^{[i]}} \mathcal{M}^{(3,0)}\left(d, a_{2},\left\{a_{l}\right\}_{3,6}^{[i]} ;\left(1-\xi_{i}\right) k_{1}, k_{2},\left\{k_{l}\right\}_{3,6}^{[i]}\right) \\
& \times \mathcal{S}_{3}([i]) d \phi_{3}\left(\left(1-\xi_{i}\right) k_{1}, k_{2} \rightarrow\left\{k_{l}\right\}_{3,6}^{[i]}\right) d \xi_{i},
\end{aligned}
$$

since the dependence upon the index $i$ is immaterial. Eq. (4.50) is of the same form of eq. (4.46). It is now apparent that the quantity

$$
d \hat{\sigma}_{a_{1} a_{2}, i}^{(i n,+)}=d \sigma_{a_{1} a_{2}, i}^{(i n,+)}+\frac{1}{4} d \sigma_{a_{1} a_{2}}^{(c n t+)}
$$


does not contain any purely collinear pole (i.e., proportional to the Altarelli-Parisi kernel for $z<1$ ). Expanding in a Taylor series eq. (4.51), and using

$$
P_{a b}^{<}(z, \epsilon)=P_{a b}^{<}(z, 0)+\epsilon P_{a b}^{\prime<}(z, 0),
$$

we have

$$
\begin{aligned}
d \hat{\sigma}_{a_{1} a_{2}, i}^{(i n,+)}= & \frac{\alpha_{S}}{2 \pi} \frac{1}{\bar{\epsilon}}\left(\gamma\left(a_{1}\right)+2 C\left(a_{1}\right) \log \xi_{c u t}\right) \\
& \times \frac{1}{4 !} \sum_{\left\{a_{l}\right\}_{3,6}^{[i]}} \mathcal{M}^{(3,0)}\left(\left\{a_{l}\right\}_{1,6}^{[i]} ;\left\{k_{l}\right\}_{1,6}^{[i]}\right) \mathcal{S}_{3}([i]) d \phi_{3}\left(k_{1}, k_{2} \rightarrow\left\{k_{l}\right\}_{3,6}^{[i]}\right) \\
+ & \frac{\alpha_{S}}{2 \pi} \sum_{d}\left\{\xi_{i} P_{d a_{1}}^{<}\left(1-\xi_{i}, 0\right)\left[\left(\frac{1}{\xi_{i}}\right)_{c} \log \frac{S \delta_{I}}{2 \mu^{2}}+2\left(\frac{\log \xi_{i}}{\xi_{i}}\right)_{c}\right]\right. \\
& \times \frac{1}{4 !} \sum_{\left\{a_{l}\right\}_{3,6}^{[i]}} \mathcal{M}^{(3,0)}\left(d, a_{2},\left\{a_{l}\right\}_{3,6}^{[i]} ;\left(1-\xi_{i a_{1}}\left(1-\xi_{i}, 0\right)\left(\frac{1}{\xi_{i}}\right)_{c}-K_{d a_{1}}\left(1-k_{2},\left\{k_{l}\right\}_{3,6}^{[i]}\right)\right.\right. \\
& \times \mathcal{S}_{3}([i]) d \phi_{3}\left(\left(1-\xi_{i}\right) k_{1}, k_{2} \rightarrow\left\{k_{l}\right\}_{3,6}^{[i]}\right) d \xi_{i} .
\end{aligned}
$$

The pole part of this equation, which originates from the $\delta$ term in the flavour-diagonal Altarelli-Parisi kernels, cancels a corresponding term in the soft-virtual contribution. The remaining part is finite, and can be numerically evaluated. The dependence upon the index $i$ in the RHS of eq. (4.53) is trivial, and the sum over $i$ is very easily carried out. After some algebra, we get

$$
\begin{aligned}
d \hat{\sigma}_{a_{1} a_{2}}^{(i n,+)}= & \sum_{i} d \hat{\sigma}_{a_{1} a_{2}, i}^{(i n,+)} \\
= & \frac{\alpha_{S}}{2 \pi}\left(\frac{(4 \pi)^{\epsilon}}{\Gamma(1-\epsilon)}\left(\frac{\mu^{2}}{Q^{2}}\right)^{\epsilon} \frac{1}{\epsilon}-\log \frac{\mu^{2}}{Q^{2}}\right) \\
& \times\left(\gamma\left(a_{1}\right)+2 C\left(a_{1}\right) \log \xi_{c u t}\right) \sum_{\left\{a_{l}\right\}_{3,5}} d \sigma^{(0)}\left(\left\{a_{l}\right\}_{1,5} ;\left\{k_{l}\right\}_{1,5}\right) \\
+ & \frac{\alpha_{S}}{2 \pi} \sum_{d}\left\{\xi P_{d a_{1}}^{<}(1-\xi, 0)\left[\left(\frac{1}{\xi}\right)_{c} \log \frac{S \delta_{I}}{2 \mu^{2}}+2\left(\frac{\log \xi}{\xi}\right)_{c}\right]\right. \\
& \times \sum_{\left\{a_{l}\right\}_{3,5}} d \sigma^{(0)}\left(d, a_{2},\left\{a_{l}\right\}_{3,5} ;(1-\xi) k_{1}, k_{2},\left\{k_{l}\right\}_{3,5}\right) d \xi
\end{aligned}
$$


where $d \sigma^{(0)}$ was defined in eq. (4.28). In the very same way, we have

$$
\begin{aligned}
d \hat{\sigma}_{a_{1} a_{2}}^{(i n,-)}= & \frac{\alpha_{S}}{2 \pi}\left(\frac{(4 \pi)^{\epsilon}}{\Gamma(1-\epsilon)}\left(\frac{\mu^{2}}{Q^{2}}\right)^{\epsilon} \frac{1}{\epsilon}-\log \frac{\mu^{2}}{Q^{2}}\right) \\
& \times\left(\gamma\left(a_{2}\right)+2 C\left(a_{2}\right) \log \xi_{c u t}\right) \sum_{\left\{a_{l}\right\}_{3,5}} d \sigma^{(0)}\left(\left\{a_{l}\right\}_{1,5} ;\left\{k_{l}\right\}_{1,5}\right) \\
+ & \frac{\alpha_{S}}{2 \pi} \sum_{d}\left\{\xi P_{d a_{2}}^{<}(1-\xi, 0)\left[\left(\frac{1}{\xi}\right)_{c} \log \frac{S \delta_{I}}{2 \mu^{2}}+2\left(\frac{\log \xi}{\xi}\right)_{c}\right]\right. \\
& \left.\quad-\xi P_{d a_{2}}^{\prime<}(1-\xi, 0)\left(\frac{1}{\xi}\right)_{c}-K_{d a_{2}}(1-\xi)\right\} \\
& \times \sum_{\left\{a_{l}\right\}_{3,5}} d \sigma^{(0)}\left(a_{1}, d,\left\{a_{l}\right\}_{3,5} ; k_{1},(1-\xi) k_{2},\left\{k_{l}\right\}_{3,5}\right) d \xi
\end{aligned}
$$

\subsection{Final state singularities}

We finally turn to the problem of regulating the divergencies appearing in eq. (4.15). To this purpose, we rewrite eq. (4.3) in the following way

$$
k_{i}=\frac{\sqrt{S}}{2} \xi_{i}\left(1, \hat{k}_{i}\right), \hat{k}_{i}=\hat{p} R, \hat{p}=(\overrightarrow{0}, 1) .
$$

Here $\hat{p}$ is a $3-2 \epsilon$ dimensional vector and $R$ a $3-2 \epsilon$ dimensional rotation matrix. With $\epsilon=0$ and eq. (4.3), the explicit form of $R$ can be worked out, but we will not need it in the following. We then parametrize the momentum of parton $j$ as

$$
k_{j}=\frac{\sqrt{S}}{2} \xi_{j}\left(1, \hat{k}_{j}\right), \quad \hat{k}_{j}=\hat{p}_{j} R, \hat{p}_{j}=\left(\sqrt{1-y_{j}^{2}} \vec{e}_{j T}, y_{j}\right)
$$

From this definition we get

$$
k_{i} \cdot k_{j}=\left(\frac{\sqrt{S}}{2}\right)^{2} \xi_{i} \xi_{j}\left(1-y_{j}\right) .
$$

Therefore, the collinear limit $i \| j$ is obtained with the parametrization of eq. (4.57) by letting $y_{j} \rightarrow 1$. It follows that we can regulate final state divergencies by multiplying the invariant amplitude squared by the factor $\left(1-y_{j}\right)$. We also write

$$
\begin{aligned}
d \phi & =d \tilde{\phi} d \phi(j) \\
& =d \tilde{\phi} \frac{1}{2(2 \pi)^{3-2 \epsilon}}\left(\frac{\sqrt{S}}{2}\right)^{2-2 \epsilon} \xi_{j}^{1-2 \epsilon}\left(1-y_{j}^{2}\right)^{-\epsilon} d \xi_{j} d y_{j} d \Omega_{j}^{(2-2 \epsilon)}
\end{aligned}
$$


where, from eq. (4.5),

$$
d \tilde{\phi}=(2 \pi)^{4-2 \epsilon} \delta^{4-2 \epsilon}\left(k_{1}+k_{2}-\sum_{l=3}^{6} k_{l}\right) \prod_{l}^{[i j]} \frac{d^{3-2 \epsilon} k_{l}}{(2 \pi)^{3-2 \epsilon} 2 k_{l}^{0}} .
$$

Eq. (4.15) becomes

$$
\begin{aligned}
d \sigma_{a_{1} a_{2}, i j}^{(o u t)} & =\frac{1}{4 !}\left[\left(\frac{1}{\xi_{i}}\right)_{c}-2 \epsilon\left(\frac{\log \xi_{i}}{\xi_{i}}\right)_{c}\right]\left(\left(1-y_{j}\right) \xi_{i}^{2} \sum_{\left\{a_{l}\right\}_{3,6}} \mathcal{M}^{(4)}\left(\left\{a_{l}\right\}_{1,6}\right)\right) \\
& \times \mathcal{S}_{i j}^{(1)} \theta\left(d_{j}-d_{i}\right) d \tilde{\phi}\left(\frac{1}{2(2 \pi)^{3-2 \epsilon}}\left(\frac{\sqrt{S}}{2}\right)^{2-2 \epsilon}\right)^{2}\left(1-y_{i}^{2}\right)^{-\epsilon} d \xi_{i} d y_{i} d \Omega_{i}^{(2-2 \epsilon)} \\
& \times \xi_{j}^{1-2 \epsilon}\left(1-y_{j}\right)^{-1-\epsilon}\left(1+y_{j}\right)^{-\epsilon} d \xi_{j} d y_{j} d \Omega_{j}^{(2-2 \epsilon)}
\end{aligned}
$$

We can therefore use the identity

$$
\left(1-y_{j}\right)^{-1-\epsilon}=-\frac{\left(\delta_{o}\right)^{-\epsilon}}{\epsilon} \delta\left(1-y_{j}\right)+\left(\frac{1}{1-y_{j}}\right)_{\delta_{o}}+\mathcal{O}(\epsilon)
$$

where $0<\delta_{o} \leq 2$, to split $d \sigma_{a_{1} a_{2}, i j}^{(o u t)}$ into two terms

$$
d \sigma_{a_{1} a_{2}, i j}^{(o u t)}=d \sigma_{a_{1} a_{2}, i j}^{(o u t,+)}+d \sigma_{a_{1} a_{2}, i j}^{(o u t, f)},
$$

where $d \sigma_{a_{1} a_{2}, i j}^{(o u t,+)}$ is proportional to $\delta\left(1-y_{j}\right)$ and $d \sigma_{a_{1} a_{2}, i j}^{(o u t, f)}$ is free of singularities (and therefore we can set $\epsilon=0$ ) and can be numerically integrated. Explicitly

$$
\begin{aligned}
d \sigma_{a_{1} a_{2}, i j}^{(\text {out }, f)} & =\frac{1}{4 !}\left(\frac{1}{\xi_{i}}\right)_{c}\left(\frac{1}{1-y_{j}}\right)_{\delta_{o}}\left(\left(1-y_{j}\right) \xi_{i}^{2} \xi_{j} \sum_{\left\{a_{l}\right\}_{3,6}} \mathcal{M}^{(4)}\left(\left\{a_{l}\right\}_{1,6}\right)\right) \mathcal{S}_{i j}^{(1)} \theta\left(d_{j}-d_{i}\right) \\
& \times\left(\frac{1}{2(2 \pi)^{3}}\left(\frac{\sqrt{S}}{2}\right)^{2}\right)^{2} d \tilde{\phi} d \xi_{i} d \xi_{j} d y_{i} d y_{j} d \varphi_{i} d \varphi_{j}
\end{aligned}
$$

and

$$
d \sigma_{a_{1} a_{2}, i j}^{(o u t,+)}=-\frac{\left(2 \delta_{o}\right)^{-\epsilon}}{\epsilon} \delta\left(1-y_{j}\right) d y_{j} \mathcal{D} \mathcal{A} d \mu
$$

where

$$
\begin{aligned}
& \mathcal{D}=\frac{1}{4 !}\left[\left(\frac{1}{\xi_{i}}\right)_{c}-2 \epsilon\left(\frac{\log \xi_{i}}{\xi_{i}}\right)_{c}\right] \\
& \mathcal{A}=\left(\left(1-y_{j}\right) \xi_{i}^{2} \sum_{\left\{a_{l}\right\}_{3,6}} \mathcal{M}^{(4)}\left(\left\{a_{l}\right\}_{1,6}\right)\right) \mathcal{S}_{i j}^{(1)} \theta\left(d_{j}-d_{i}\right),
\end{aligned}
$$




$$
d \mu=\left(\frac{1}{2(2 \pi)^{3-2 \epsilon}}\left(\frac{\sqrt{S}}{2}\right)^{2-2 \epsilon}\right)^{2} d \tilde{\phi} \xi_{j}^{1-2 \epsilon}\left(1-y_{i}^{2}\right)^{-\epsilon} d \xi_{i} d \xi_{j} d y_{i} d \Omega_{i}^{(2-2 \epsilon)} d \Omega_{j}^{(2-2 \epsilon)}
$$

We perform the following change of variables

$$
\xi_{i}=(1-z) \xi_{7}, \quad \xi_{j}=z \xi_{7}, \quad \Rightarrow d \xi_{i} d \xi_{j}=\xi_{7} d \xi_{7} d z
$$

With this definition, we have

$$
\delta\left(1-y_{j}\right)\left(k_{i}+k_{j}\right) \equiv \delta\left(1-y_{j}\right) k_{7}=\delta\left(1-y_{j}\right) \frac{\sqrt{S}}{2} \xi_{7}\left(1, \sqrt{1-y_{i}^{2}} \vec{e}_{i T}, y_{i}\right),
$$

which defines the momentum $k_{7}$; notice that the definition of $\xi_{7}$ is therefore consistent with eq. (4.3); in the collinear limit, this parameter is proportional to the energy of the parton which eventually splits into the two collinear partons $i$ and $j$. With the definition of $z$ in eq. (4.70) we have, analogously to eq. 4.38),

$$
\begin{aligned}
\lim _{y_{j} \rightarrow 1} \mathcal{M}^{(4)}\left(\left\{a_{l}\right\}_{1,6} ;\left\{k_{l}\right\}_{1,6}\right) & =\frac{4 \pi \alpha_{S} \mu^{2 \epsilon}}{k_{i} \cdot k_{j}} \Delta\left(\left\{a_{l}\right\}_{1,6} ;\left\{k_{l}\right\}_{1,6}\right) \\
& +\frac{4 \pi \alpha_{S} \mu^{2 \epsilon}}{k_{i} \cdot k_{j}} P_{a_{j} a_{7}}^{<}(z, \epsilon) \mathcal{M}^{(3,0)}\left(\left\{a_{l}\right\}_{1,7}^{[i j]} ;\left\{k_{l}\right\}_{1,7}^{[i j]}\right)
\end{aligned}
$$

with

$$
a_{7}=S\left(a_{i}, a_{j}\right)
$$

Using eqs. (4.72), 2.56) and (2.59) we get

$$
\begin{aligned}
\lim _{y_{j} \rightarrow 1} \mathcal{A} & =4 \pi \alpha_{S} \mu^{2 \epsilon}\left(\frac{\sqrt{S}}{2}\right)^{-2} \frac{1-z}{z} \theta\left(z-\frac{1}{2}\right) \mathcal{S}_{3}([i j]) \\
& \times \sum_{\left\{a_{l}\right\}_{3,6}}\left[P_{a_{j} a_{7}}^{<}(z, \epsilon) \mathcal{M}^{(3,0)}\left(\left\{a_{l}\right\}_{1,7}^{[i j]} ;\left\{k_{l}\right\}_{1,7}^{[i j]}\right)+\Delta\left(\left\{a_{l}\right\}_{1,6} ;\left\{k_{l}\right\}_{1,6}\right)\right],
\end{aligned}
$$

since from eq. (4.70) immediately follows

$$
\theta\left(d_{j}-d_{i}\right) \equiv \theta\left(z-\frac{1}{2}\right) .
$$

We can now exploit the following identities

$$
\begin{aligned}
\left(\frac{1}{\xi_{i}}\right)_{c} & =\frac{1}{\xi_{7}} \mathcal{D}^{(0)}(z), \\
\left(\frac{\log \xi_{i}}{\xi_{i}}\right)_{c} & =\frac{1}{\xi_{7}} \mathcal{D}^{(1)}(z),
\end{aligned}
$$


where

$$
\begin{aligned}
& \mathcal{D}^{(0)}(z)=\left(\frac{1}{1-z}\right)_{+}+\log \left(\frac{\xi_{7}}{\xi_{\text {cut }}}\right) \delta(1-z) \\
& \mathcal{D}^{(1)}(z)=\left(\frac{\log (1-z)}{1-z}\right)_{+}+\log \xi_{7}\left(\frac{1}{1-z}\right)_{+}+\frac{1}{2}\left(\log ^{2} \xi_{7}-\log ^{2} \xi_{\text {cut }}\right) \delta(1-z) .
\end{aligned}
$$

Finally, with the change of variables in eq. (4.70), the measure becomes

$$
d \mu=\left(\frac{1}{2(2 \pi)^{3-2 \epsilon}}\left(\frac{\sqrt{S}}{2}\right)^{2-2 \epsilon}\right)^{2} d \tilde{\phi} z^{1-2 \epsilon} d z \xi_{7}^{2-2 \epsilon} d \xi_{7}\left(1-y_{i}^{2}\right)^{-\epsilon} d y_{i} d \Omega_{i}^{(2-2 \epsilon)} d \Omega_{j}^{(2-2 \epsilon)} .
$$

After some algebra, and using the analogous of eqs. (4.41) and (4.42) when integrating over $d \Omega_{j}^{(2-2 \epsilon)}$, we get

$$
\begin{aligned}
d \sigma_{a_{1} a_{2}, i j}^{(o u t,+)} & =-\frac{\left(2 \delta_{o}\right)^{-\epsilon}}{\epsilon} \frac{2 \pi^{1-\epsilon}}{\Gamma(1-\epsilon)} 4 \pi \alpha_{S} \mu^{2 \epsilon}\left(\frac{\sqrt{S}}{2}\right)^{-2} \frac{1}{4 !}\left[\mathcal{D}^{(0)}(z)-2 \epsilon \mathcal{D}^{(1)}(z)\right] \theta\left(z-\frac{1}{2}\right) \\
& \times d z(1-z) z^{-2 \epsilon} \sum_{\left\{a_{l}\right\}_{3,6}} P_{a_{j} a_{7}}^{<}(z, \epsilon) \mathcal{M}^{(3,0)}\left(\left\{a_{l}\right\}_{1,7}^{[i j]} ;\left\{k_{l}\right\}_{1,7}^{[i j]}\right) \mathcal{S}_{3}([i j]) \\
& \times\left(\frac{1}{2(2 \pi)^{3-2 \epsilon}}\left(\frac{\sqrt{S}}{2}\right)^{2-2 \epsilon}\right)^{2} d \tilde{\phi} \xi_{7}^{1-2 \epsilon} d \xi_{7}\left(1-y_{i}^{2}\right)^{-\epsilon} d y_{i} d \Omega_{i}^{(2-2 \epsilon)}
\end{aligned}
$$

Using eq. (4.71), we can see that the following relation holds

$$
d \phi_{3}\left(k_{1}, k_{2} \rightarrow\left\{k_{l}\right\}_{3,7}^{[i j]}\right)=\frac{1}{2(2 \pi)^{3-2 \epsilon}}\left(\frac{\sqrt{S}}{2}\right)^{2-2 \epsilon} \xi_{7}^{1-2 \epsilon}\left(1-y_{i}^{2}\right)^{-\epsilon} d \xi_{7} d y_{i} d \Omega_{i}^{(2-2 \epsilon)} d \tilde{\phi}
$$

Therefore we have

$$
\begin{aligned}
d \sigma_{a_{1} a_{2}, i j}^{(o u t,+)} & =-\frac{\alpha_{S}}{2 \pi}\left(\frac{1}{\bar{\epsilon}}-\log \frac{S \delta_{o}}{2 \mu^{2}}\right) \sum_{\left\{a_{l}\right\}_{3,6}}\left[\mathcal{I}_{a_{j} a_{7}}^{(0)}-2 \epsilon \mathcal{I}_{a_{j} a_{7}}^{(1)}\right] \\
& \times \frac{1}{4 !} \mathcal{M}^{(3,0)}\left(\left\{a_{l}\right\}_{1,7}^{[i j]} ;\left\{k_{l}\right\}_{1,7}^{[i j]}\right) \mathcal{S}_{3}([i j]) d \phi_{3}\left(k_{1}, k_{2} \rightarrow\left\{k_{l}\right\}_{3,7}^{[i j]}\right)
\end{aligned}
$$

where

$$
\begin{aligned}
& \mathcal{I}_{a b}^{(0)}=\int_{0}^{1} d z z^{-2 \epsilon}(1-z) P_{a b}^{<}(z, \epsilon) \theta\left(z-\frac{1}{2}\right) \mathcal{D}^{(0)}(z), \\
& \mathcal{I}_{a b}^{(1)}=\int_{0}^{1} d z z^{-2 \epsilon}(1-z) P_{a b}^{<}(z, \epsilon) \theta\left(z-\frac{1}{2}\right) \mathcal{D}^{(1)}(z) .
\end{aligned}
$$


Eq. (4.83) shows that the singular term due to final state collinear emission factorizes into the product of two quantities; a cross section, which has a two-to-three partonic kinematics, and a term dependent upon the Altarelli-Parisi kernels, which completely describes the collinear splitting. We can sum over $i, j$ the quantities $d \sigma_{a_{1} a_{2}, i j}^{(o u t,+)}$. It is a simple matter of combinatorial calculus to see that this sum can be rewritten in the following way, by simply relabeling the partons

$$
d \sigma_{a_{1} a_{2}}^{(\text {out },+)}=\sum_{i} \sum_{j}^{[i]} d \sigma_{a_{1} a_{2}, i j}^{(o u t,+)}=\sum_{j=3}^{5} d \tilde{\sigma}_{a_{1} a_{2}, j}^{(o u t,+)}
$$

where

$$
d \tilde{\sigma}_{a_{1} a_{2}, j}^{(o u t,+)}=-\frac{\alpha_{S}}{2 \pi}\left(\frac{1}{\bar{\epsilon}}-\log \frac{S \delta_{o}}{2 \mu^{2}}\right) \sum_{\left\{a_{l}\right\}_{3,5}} \sum_{d}\left[\mathcal{I}_{d a_{j}}^{(0)}-2 \epsilon \mathcal{I}_{d a_{j}}^{(1)}\right] d \sigma^{(0)}\left(\left\{a_{l}\right\}_{1,5}\right) .
$$

The expression for $\sum_{d}\left[\mathcal{I}_{d a_{j}}^{(0)}-2 \epsilon \mathcal{I}_{d a_{j}}^{(1)}\right]$ is given in appendix A. By substituting it in eq. (4.87) we get

$$
\begin{aligned}
d \sigma_{a_{1} a_{2}}^{(\text {out },+)}= & \frac{\alpha_{S}}{2 \pi} \frac{(4 \pi)^{\epsilon}}{\Gamma(1-\epsilon)}\left(\frac{\mu^{2}}{Q^{2}}\right)^{\epsilon} \sum_{\left\{a_{l}\right\}_{3,5}} d \sigma^{(0)}\left(\left\{a_{l}\right\}_{1,5}\right) \\
& \times \frac{1}{\epsilon} \sum_{j=3}^{5}\left[\gamma\left(a_{j}\right)-2 C\left(a_{j}\right) \log \frac{2 E_{j}}{\xi_{\text {cut }} \sqrt{S}}\right] \\
+ & \frac{\alpha_{S}}{2 \pi} \sum_{\left\{a_{l}\right\}_{3,5}} d \sigma^{(0)}\left(\left\{a_{l}\right\}_{1,5}\right) \\
& \times \sum_{j=3}^{5}\left[\gamma^{\prime}\left(a_{j}\right)-\log \frac{S \delta_{o}}{2 Q^{2}}\left(\gamma\left(a_{j}\right)-2 C\left(a_{j}\right) \log \frac{2 E_{j}}{\xi_{c u t} \sqrt{S}}\right)\right. \\
& \left.+2 C\left(a_{j}\right)\left(\log ^{2} \frac{2 E_{j}}{\sqrt{S}}-\log ^{2} \xi_{\text {cut }}\right)-2 \gamma\left(a_{j}\right) \log \frac{2 E_{j}}{\sqrt{S}}\right] .
\end{aligned}
$$

The explicit expression for $\gamma^{\prime}\left(a_{j}\right)$ is given in eqs. (A.12) and (A.13).

\section{Results}

In the previous sections we have shown that the subtracted next-to-leading order contribution, defined in eq. (2.5), can be written as the sum of a term with a two-tofour partonic kinematics, and a term with a two-to-three partonic kinematics

$$
d \hat{\sigma}_{a_{1} a_{2}}^{(1)}=d \hat{\sigma}_{a_{1} a_{2}}^{(1,4 p)}+d \hat{\sigma}_{a_{1} a_{2}}^{(1,3 p)}
$$


The two-to-four part is

$$
d \hat{\sigma}_{a_{1} a_{2}}^{(1,4 p)}=\sum_{i}\left(d \sigma_{a_{1} a_{2}, i}^{(f i n)}+d \sigma_{a_{1} a_{2}, i}^{(i n, f)}+\sum_{j}^{[i]} d \sigma_{a_{1} a_{2}, i j}^{(o u t, f)}\right)
$$

where the quantities appearing in the RHS of this equation were given in eqs. (2.64), 4.37) and (4.65) respectively. The two-to-three part is

$$
d \hat{\sigma}_{a_{1} a_{2}}^{(1,3 p)}=d \sigma_{a_{1} a_{2}}^{(v)}+d \sigma_{a_{1} a_{2}}^{(s)}+d \hat{\sigma}_{a_{1} a_{2}}^{(i n,+)}+d \hat{\sigma}_{a_{1} a_{2}}^{(i n,-)}+d \sigma_{a_{1} a_{2}}^{(o u t,+)}
$$

where the quantities appearing in the RHS of this equation were given in eqs. (2.15) (with the matrix element of eq. (3.1)), (4.27), (4.54), (4.55) and (4.88). Every term in the RHS of eq. (5.2) is finite, and can be numerically integrated. On the other hand, the quantities in the RHS of eq. (5.3) are divergent; nevertheless, from their explicit expression previously reported, it is apparent that the divergencies cancel in the sum, and $d \hat{\sigma}_{a_{1} a_{2}}^{(1,3 p)}$ is finite. The finite part of eqs. (4.54) and (4.55), due to initial state collinear contribution, is slightly more complicated than the usual twoto-three kinematics contribution, since it has an additional folding in the variable $\xi$. Therefore, we further split eq. (5.3) into a sum of two terms, one of which does not contain any folding. The reason for doing so is that in a numerical computation the two terms have to be differently treated, in spite of the fact of having the same final state kinematics. We have

$$
d \hat{\sigma}_{a_{1} a_{2}}^{(1,3 p)}=d \hat{\sigma}_{a_{1} a_{2}}^{(1,3 p v)}+d \hat{\sigma}_{a_{1} a_{2}}^{(1,3 p r)}
$$

The part without $\xi$ folding is

$$
\begin{aligned}
d \hat{\sigma}_{a_{1} a_{2}}^{(1,3 p v)} & =\frac{\alpha_{S}}{2 \pi} \sum_{\left\{a_{l}\right\}_{3,5}} \mathcal{Q}\left(\left\{a_{l}\right\}_{1,5}\right) d \sigma^{(0)}\left(\left\{a_{l}\right\}_{1,5}\right) \\
& +\frac{\alpha_{S}}{2 \pi} \frac{1}{2} \sum_{\substack{n, m=1 \\
n \neq m}}^{5} \mathcal{I}_{m n}^{(r e g)} \sum_{\left\{a_{l}\right\}_{3,5}} d \sigma_{m n}^{(0)}\left(\left\{a_{l}\right\}_{1,5}\right) \\
& +\frac{\alpha_{S}}{2 \pi} \frac{1}{3 !} \sum_{\left\{a_{l}\right\}_{3,5}} \mathcal{M}_{N S}^{(3,1)}\left(\left\{a_{l}\right\}_{1,5}\right) \mathcal{S}_{3} d \phi_{3}
\end{aligned}
$$


where

$$
\begin{aligned}
\mathcal{Q}\left(\left\{a_{l}\right\}_{1,5}\right)= & \sum_{j=3}^{5}\left[\gamma^{\prime}\left(a_{j}\right)-\log \frac{S \delta_{o}}{2 Q^{2}}\left(\gamma\left(a_{j}\right)-2 C\left(a_{j}\right) \log \frac{2 E_{j}}{\xi_{c u t} \sqrt{S}}\right)\right. \\
& \left.+2 C\left(a_{j}\right)\left(\log ^{2} \frac{2 E_{j}}{\sqrt{S}}-\log ^{2} \xi_{c u t}\right)-2 \gamma\left(a_{j}\right) \log \frac{2 E_{j}}{\sqrt{S}}\right] \\
- & \log \frac{\mu^{2}}{Q^{2}}\left(\gamma\left(a_{1}\right)+2 C\left(a_{1}\right) \log \xi_{\text {cut }}+\gamma\left(a_{2}\right)+2 C\left(a_{2}\right) \log \xi_{\text {cut }}\right),
\end{aligned}
$$

and $d \sigma^{(0)}, d \sigma_{m n}^{(0)}$ were defined in eqs. 4.28 ) and (4.26) respectively. The part with the folding can directly be read from eqs. (4.54) and (4.55), and it is

$$
\begin{aligned}
d \hat{\sigma}_{a_{1} a_{2}}^{(1,3 p r)=} & \frac{\alpha_{S}}{2 \pi} \sum_{d}\left\{\xi P_{d a_{1}}^{<}(1-\xi, 0)\left[\left(\frac{1}{\xi}\right)_{c} \log \frac{S \delta_{I}}{2 \mu^{2}}+2\left(\frac{\log \xi}{\xi}\right)_{c}\right]_{c}\right. \\
& \left.-\xi P_{d a_{1}}^{<<}(1-\xi, 0)\left(\frac{1}{\xi}\right)_{c}-K_{d a_{1}}(1-\xi)\right\} \\
& \times \sum_{\left\{a_{l}\right\}_{3,5}} d \sigma^{(0)}\left(d, a_{2},\left\{a_{l}\right\}_{3,5} ;(1-\xi) k_{1}, k_{2},\left\{k_{l}\right\}_{3,5}\right) d \xi \\
+ & \frac{\alpha_{S}}{2 \pi} \sum_{d}\left\{\xi P_{d a_{2}}^{<}(1-\xi, 0)\left[\left(\frac{1}{\xi}\right)_{c} \log \frac{S \delta_{I}}{2 \mu^{2}}+2\left(\frac{\log \xi}{\xi}\right)_{c}\right]\right. \\
& \left.\quad-\xi P_{d a_{2}}^{\prime<}(1-\xi, 0)\left(\frac{1}{\xi}\right)_{c}-K_{d a_{2}}(1-\xi)\right\} \\
& \times \sum_{\left\{a_{l}\right\}_{3,5}} d \sigma^{(0)}\left(a_{1}, d,\left\{a_{l}\right\}_{3,5} ; k_{1},(1-\xi) k_{2},\left\{k_{l}\right\}_{3,5}\right) d \xi .
\end{aligned}
$$

We have derived our results in the partonic center-of-mass frame. This slightly constrains the general validity of the formalism, since in the numerical computations $x_{1}$ and $x_{2}$, the Bjorken $x$ of the incoming partons, have to be chosen as independent integration variables. Notice that they define the boost from the hadronic center-ofmass frame to the partonic one. We stress that this constraint can be very easily relaxed, without affecting the correctness of the derivation. Nevertheless, as far as the numerical computations are concerned, it appears to be advantageous to work in the partonic center-of-mass frame. We remind that in previous applications of the subtraction method to jet physics $x_{1}$ and $x_{2}$ have been expressed in terms of transverse momenta and rapidities. With our finite next-to-leading order partonic cross sections, we can get the physical cross section using eq. (2.1). 
A final remark on the numerical computation: the parameters $\xi_{\text {cut }}, \delta_{I}$ and $\delta_{O}$ define the soft and collinear subtractions. Thanks to this property, we can freely redefine the terms in the RHS of eq. (5.1) without affecting the sum. This gives us the possibility of a significant numerical check on the correctness of the calculation; the physical results have to be independent from the value of these parameters. Also, notice that a clever choice of the parameters results in saving computing time. If for example eq. (4.8) is considered, it is apparent that in most cases the soft counterterm $(f(0))$ is not evaluated if $\xi_{c u t}$ is small. On the other hand, $\xi_{\text {cut }}$ should not have a value too close to 0 , because in this way the quality of the convergence is rather poor.

\section{Conclusions}

We have studied the production of three-jet inclusive quantities at the next-toleading order in $\mathrm{QCD}$, using the subtraction method. Comparing to previous treatments [19], in which the one- or two-jet production is dealt with this method, we used a somewhat different approach in at least two important aspects. First of all, we used angle and energy variables, instead of transverse momenta and rapidities; this results in a simplification when integrating the eikonal factors, which appear whenever one of the final state partons gets soft. As a second feature, we fully exploited the measurement function, which defines infrared-safe observables, to disentangle the soft and collinear singularities in the real contribution. In fact, the measurement function can always be cast as a sum of terms, each of them getting contributions only from the infrared singular regions associated with a given parton. At the very end, we have therefore splitted the cross section into single-singular contributions, without the drawback of decomposing the transition amplitude squared with partial fractioning.

The analytical results obtained were organized in a form suited for numerical computations. We splitted the real plus virtual contribution in a sum of terms with different final state partonic kinematics. The possibility has been left to redefine

them up to a finite piece, without affecting the sum, which is the only quantity being physically meaningful.

Although we treated explicitly only three-jet production in hadron-hadron collisions, our formalism can be easily extended to $n$-jet production. Furthermore, we emphasize that it is also applicable to $n$-jet production in $e^{+} e^{-}$annihilation and in photon-hadron collisions. 


\section{APPENDIX A: Soft and collinear integrals}

In this appendix, we collect the results for the integrals of the eikonal factors, which are substituted into eq. (4.19), and for the integrals of the Altarelli-Parisi kernels, with the prescription defined in eq. (4.84) and (4.85).

- Soft integrals: given eq. (4.19), we will consider the quantities

$$
\mathcal{J}_{n m}=\int E_{n m} d \omega_{i}
$$

where

$$
\begin{aligned}
E_{n m} & =\left(\frac{\sqrt{S}}{2}\right)^{2} \frac{k_{n} \cdot k_{m}}{k_{n} \cdot k_{i} k_{m} \cdot k_{i}} \xi_{i}^{2} \\
d \omega_{i} & =\left(1-y_{i}^{2}\right)^{-\epsilon} d y_{i} d \Omega_{i}^{(2-2 \epsilon)}
\end{aligned}
$$

Using eq. (4.3) we can rewrite

$$
E_{n m}=\frac{1-\cos \theta_{n m}}{\left(1-\cos \theta_{n i}\right)\left(1-\cos \theta_{m i}\right)},
$$

where $\theta_{\alpha \beta}$ is the angle between the directions of the three-momenta $\vec{k}_{\alpha}$ and $\vec{k}_{\beta}$. The measure $d \omega_{i}$ is the angular measure in $3-2 \epsilon$ dimensions for the momentum $k_{i}$; since also the eikonal factor is rotationally invariant, we can redefine at will the angular variables of the momentum $\vec{k}_{i}$. If we choose

$$
\vec{k}_{i}=(. ., \sin \varphi \sin \theta, \cos \varphi \sin \theta, \cos \theta),
$$

then we have

$$
d \omega_{i}=2^{1-2 \epsilon} \pi^{-\epsilon} \frac{\Gamma(1-\epsilon)}{\Gamma(1-2 \epsilon)} \sin ^{-2 \epsilon} \varphi \sin ^{-2 \epsilon} \theta d \cos \theta d \varphi,
$$

where an integration has been carried out over the variables upon which the eikonal factor can not depend. Finally, we decompose eq. (A.4) as follows

$$
E_{n m}=\frac{1}{1-\cos \theta_{n i}}+\frac{1}{2}\left(E_{n m}-\frac{1}{1-\cos \theta_{n i}}-\frac{1}{1-\cos \theta_{m i}}\right)+(n \leftrightarrow m) .
$$

The first term in the RHS of this equation will give, after integration, a collinear pole. The second term is regulated by means of the subtractions, and will contribute 
a finite term to the integral. The final result is

$$
\begin{aligned}
\mathcal{J}_{n m}= & 2^{1-2 \epsilon} \pi^{-\epsilon} \frac{\Gamma(1-\epsilon)}{\Gamma(1-2 \epsilon)} \\
\times & {\left[\frac{2^{2 \epsilon} \pi \Gamma(1-2 \epsilon)}{(\Gamma(1-\epsilon))^{2}}\left(-\frac{2}{\epsilon}+4 \log 2+2 \epsilon\left(\frac{\pi^{2}}{6}-2 \log ^{2} 2\right)\right)+2 \pi \log \left(\frac{1-\cos \theta_{n m}}{2}\right)\right.} \\
& -2 \epsilon \pi\left(-\operatorname{Li}_{2}\left(\frac{1-\cos \theta_{n m}}{2}\right)+\frac{1}{2} \log ^{2}\left(2\left(1-\cos \theta_{n m}\right)\right)+\frac{\pi^{2}}{6}-2 \log ^{2} 2\right) \\
& \left.+2 \epsilon \pi \log \left(2\left(1+\cos \theta_{n m}\right)\right) \log \left(\frac{1-\cos \theta_{n m}}{2}\right)\right] .
\end{aligned}
$$

It is just a matter of trivial algebra to get from this equation the expressions of eqs. (4.22) and (4.23).

- Collinear integrals: the calculation of the integrals defined in eqs. (4.84) and (4.85) is a lengthy but trivial operation. Using eqs. (4.78) and (4.79), and taking into account that the relabeling of the partons amounts to the substitution $\xi_{7} \rightarrow \xi_{j}$ in the expressions of $\mathcal{I}_{d a_{j}}^{(0)}$ and $\mathcal{I}_{d a_{j}}^{(1)}$, and that by construction

$$
\xi_{j}=\frac{2 E_{j}}{\sqrt{S}}
$$

we get

$$
\mathcal{Z}\left(a_{j}\right)=\sum_{d}\left[\mathcal{I}_{d a_{j}}^{(0)}-2 \epsilon \mathcal{I}_{d a_{j}}^{(1)}\right]
$$

where

$$
\begin{aligned}
\mathcal{Z}\left(a_{j}\right) & =2 C\left(a_{j}\right) \log \frac{2 E_{j}}{\xi_{c u t} \sqrt{S}}-\gamma\left(a_{j}\right) \\
& -\epsilon\left[\gamma^{\prime}\left(a_{j}\right)-2 \gamma\left(a_{j}\right) \log \frac{2 E_{j}}{\sqrt{S}}+2 C\left(a_{j}\right)\left(\log ^{2} \frac{2 E_{j}}{\sqrt{S}}-\log ^{2} \xi_{\text {cut }}\right)\right],
\end{aligned}
$$

with

$$
\begin{aligned}
\gamma^{\prime}(g) & =\frac{67}{9} C_{A}-\frac{2 \pi^{2}}{3} C_{A}-\frac{23}{9} T_{F} N_{f} \\
\gamma^{\prime}(q) & =\frac{13}{2} C_{F}-\frac{2 \pi^{2}}{3} C_{F}
\end{aligned}
$$




\section{APPENDIX B: Collinear limits}

In section 4 we have stated that the $\Delta$ term of eqs. (4.38) and (4.72) does not contribute to the result, since its average over the azimuthal angle of the collinearly emitted parton is zero. This fact can be proved in $4-2 \epsilon$ dimensions studying the collinear limit in terms of the Sudakov variables (see e.g. ref. [19]). In spite of the fact that the contribution of $\Delta$ is zero, it is of some importance to know its explicit form, since it appears in local subtraction terms where it improves the numerical treatment of equations like, for example, eq. (4.37). The goal of this appendix is to express $\Delta$ as a function of the helicity amplitudes known from the literature. We will work in 4 dimensions, since we will eventually use $\Delta$ only in numerical computations.

In the following, we will consider the production process of $n$ particles

$$
1+2 \rightarrow X+i+j
$$

in the limit in which the two massless particles $i$ and $j$ become collinear to each other or collinear to the incoming massless particles 1 or 2 . Notice that the properties of the $n-2$ particles denoted collectively by $X$ are unspecified and of no importance in what follows. This implies that the results of this section are completely general and their validity is not restricted to jet physics.

We begin by considering the final state emission, that is the case in which $i$ and $j$ are collinear. Therefore, we study the structure of the process

$$
P \rightarrow i j
$$

We have to consider only three cases:
a) $g \rightarrow g g$
b) $g \rightarrow q \bar{q}$
c) $q \rightarrow q g$,

where in case c) $q$ can be either a quark or an antiquark. We begin with case a). Giving color indices $b$ and $c$ to the splitted gluons $i$ and $j$, and defining the fraction of momentum $z$ through the equations

$$
k_{i}=z k_{P}, \quad k_{j}=(1-z) k_{P}
$$




\begin{tabular}{|c||c|c|c|}
\hline$\left(h, h_{a}, h_{b}\right)$ & $g \rightarrow g g$ & $g \rightarrow q \bar{q}$ & $q \rightarrow q g$ \\
\hline \hline$(+,+,+)$ & {$[a b]$} & 0 & $z^{1 / 2}[a b]$ \\
\hline$(+,+,-)$ & $-z^{2}<a b>$ & $z^{1 / 2}(1-z)^{3 / 2}<a b>$ & $-z^{3 / 2}<a b>$ \\
\hline$(+,-,+)$ & $-(1-z)^{2}<a b>$ & $-z^{3 / 2}(1-z)^{1 / 2}<a b>$ & 0 \\
\hline$(+,-,-)$ & 0 & 0 & 0 \\
\hline$(-,+,+)$ & 0 & 0 & 0 \\
\hline$(-,+,-)$ & $(1-z)^{2}[a b]$ & $z^{3 / 2}(1-z)^{1 / 2}[a b]$ & 0 \\
\hline$(-,-,+)$ & $z^{2}[a b]$ & $-z^{1 / 2}(1-z)^{3 / 2}[a b]$ & $z^{3 / 2}[a b]$ \\
\hline$(-,-,-)$ & $-<a b>$ & 0 & $-z^{1 / 2}<a b>$ \\
\hline
\end{tabular}

Table 1: $\quad$ Splitting process $P(h) \rightarrow a\left(h_{a}\right) b\left(h_{b}\right)$ for all the possible choices of the partons $a$ and $b$ and the helicities $h, h_{a}$ and $h_{b}$. The splitting functions $S_{a P}^{h h_{a} h_{b}}$ are obtained by dividing the entries of the table by $<a b>$ $[a b] \sqrt{z(1-z)}$. By construction, the parton a has the fraction $z$ of the momentum of the parton $P$. The splitting functions $S_{g q}$ can be obtained from $S_{q q}$ with the formal substitution $z \rightarrow 1-z$.

the $n$-particle transition amplitude can be written as

$$
\mathcal{A}^{(n)}\left(h_{i}, h_{j},\left\{h_{l}\right\}\right) \stackrel{i \| j}{\longrightarrow} g_{S} \sum_{d_{e}} \sum_{h_{e}} C\left(d_{e}, b, c\right) S_{g g}^{h_{e} h_{i} h_{j}}(z) \mathcal{A}_{d_{e}}^{(n-1)}\left(h_{e},\left\{h_{l}\right\}\right) .
$$

Although the structure of this equation remains valid at all orders in perturbation theory, we will restrict in the following to the leading-order splitting. Therefore, $C\left(d_{e}, b, c\right)$ is the color factor of the $g g g$ vertex, $S_{g g}^{h_{e} h_{i} h_{j}}$ is the leading-order splitting function for fixed helicities (which can be obtained from the entries of table 1 as explained in the caption) and $\mathcal{A}_{d_{e}}^{(n-1)}$ is the transition amplitude for the process $1+2 \rightarrow$ $X+g$, when the emitted gluon has color $d_{e}$. The dependence upon the color indices $\left\{d_{l}\right\}$ of the remaining $n-2$ particles is not explicitly indicated in $\mathcal{A}^{(n-1)}$. In the following, we will systematically suppress the indication of the dependence upon all color labels with the exception of $d_{e}$, which is the only one relevant for our purposes. The quantity $\left\{h_{l}\right\}$ is the set of the helicities of the $n-2$ particles which are not involved in the splitting process. Notice that we only consider the range $z<1$, where the splitting functions with fixed helicities are meaningful. Squaring eq. (B.7) and 
summing over the color of the emitted gluon we get

$$
\begin{aligned}
\left|\mathcal{A}^{(n)}\left(h_{i}, h_{j},\left\{h_{l}\right\}\right)\right|^{2} & \stackrel{i \| j}{\longrightarrow} g_{S}^{2} \sum_{b, b^{\prime}} \sum_{c, c^{\prime}} \delta_{b b^{\prime}} \delta_{c c^{\prime}} \sum_{d_{e}, d_{e}^{\prime}} \sum_{h_{e}, h_{e}^{\prime}} C\left(d_{e}, b, c\right) C^{*}\left(d_{e}^{\prime}, b^{\prime}, c^{\prime}\right) \\
& \times \quad S_{g g}^{h_{e} h_{i} h_{j}}(z)\left(S_{g g}^{h_{e}^{\prime} h_{i} h_{j}}(z)\right)^{*} \\
& \times \quad \mathcal{A}_{d_{e}}^{(n-1)}\left(h_{e},\left\{h_{l}\right\}\right)\left(\mathcal{A}_{d_{e}^{\prime}}^{(n-1)}\left(h_{e}^{\prime},\left\{h_{l}\right\}\right)\right)^{*} .
\end{aligned}
$$

Since $C(a, b, c)=f_{a b c}$, and using the normalization conventions of Mangano and Parke [18], we have

$$
\sum_{b, b^{\prime}} \sum_{c, c^{\prime}} \delta_{b b^{\prime}} \delta_{c c^{\prime}} C\left(d_{e}, b, c\right) C^{*}\left(d_{e}^{\prime}, b^{\prime}, c^{\prime}\right)=2 C_{A} \delta_{d_{e} d_{e}^{\prime}}
$$

Therefore, eq. (B.8) becomes

$$
\begin{array}{r}
\left|\mathcal{A}^{(n)}\left(h_{i}, h_{j},\left\{h_{l}\right\}\right)\right|^{2} \stackrel{i \|_{j}}{\longrightarrow} 2 g_{S}^{2} C_{A} \sum_{d_{e}}\left(\sum_{h_{e}} S_{g g}^{h_{e} h_{i} h_{j}}(z) \mathcal{A}_{d_{e}}^{(n-1)}\left(h_{e},\left\{h_{l}\right\}\right)\right) \\
\times\left(\sum_{h_{e}^{\prime}} S_{g g}^{h_{e}^{\prime} h_{i} h_{j}}(z) \mathcal{A}_{d_{e}}^{(n-1)}\left(h_{e}^{\prime},\left\{h_{l}\right\}\right)\right)^{*} .
\end{array}
$$

Writing explicitly the sums over $h_{e}$ and $h_{e}^{\prime}$ and carrying out the multiplication we obtain

$$
\left|\mathcal{A}^{(n)}\left(h_{i}, h_{j},\left\{h_{l}\right\}\right)\right|^{2} \stackrel{i \| j}{\longrightarrow}\left|\mathcal{N}^{(n)}\left(h_{i}, h_{j},\left\{h_{l}\right\}\right)\right|^{2}+\mathcal{R}\left(h_{i}, h_{j},\left\{h_{l}\right\}\right),
$$

where

$$
\begin{aligned}
\left|\mathcal{N}^{(n)}\left(h_{i}, h_{j},\left\{h_{l}\right\}\right)\right|^{2} & =2 g_{S}^{2} C_{A}\left|S_{g g}^{+h_{i} h_{j}}(z)\right|^{2} \sum_{d_{e}}\left|\mathcal{A}_{d_{e}}^{(n-1)}\left(+,\left\{h_{l}\right\}\right)\right|^{2} \\
& +2 g_{S}^{2} C_{A}\left|S_{g g}^{-h_{i} h_{j}}(z)\right|^{2} \sum_{d_{e}}\left|\mathcal{A}_{d_{e}}^{(n-1)}\left(-,\left\{h_{l}\right\}\right)\right|^{2} \\
\mathcal{R}\left(h_{i}, h_{j},\left\{h_{l}\right\}\right) & =4 g_{S}^{2} C_{A} \operatorname{Re}\left\{S_{g g}^{+h_{i} h_{j}}(z)\left(S_{g g}^{-h_{i} h_{j}}(z)\right)^{*}\right. \\
& \left.\times \sum_{d_{e}} \mathcal{A}_{d_{e}}^{(n-1)}\left(+,\left\{h_{l}\right\}\right)\left(\mathcal{A}_{d_{e}}^{(n-1)}\left(-,\left\{h_{l}\right\}\right)\right)^{*}\right\} .
\end{aligned}
$$

By construction, given eq. (B.7), the quantity

$$
\left|\mathcal{A}^{(n-1)}\left( \pm,\left\{h_{l}\right\}\right)\right|^{2}=\sum_{\left\{d_{l}\right\}} \sum_{d_{e}}\left|\mathcal{A}_{d_{e}}^{(n-1)}\left( \pm,\left\{h_{l}\right\}\right)\right|^{2}
$$


is the $(n-1)$-particle amplitude squared and summed over colors, for a given helicity configuration $\left( \pm,\left\{h_{l}\right\}\right)$. Therefore, taking into account

$$
\sum_{h_{i}, h_{j}}\left|S_{g g}^{+h_{i} h_{j}}(z)\right|^{2}=\sum_{h_{i}, h_{j}}\left|S_{g g}^{-h_{i} h_{j}}(z)\right|^{2}=\frac{1}{C_{A}} \frac{1}{2 k_{i} \cdot k_{j}} P_{g g}^{<}(z),
$$

where $P_{g g}^{<}$is the unpolarized Altarelli-Parisi kernel for $z<1$, from eq. (B.12) we get

$$
\sum_{h_{i}, h_{j},\left\{h_{l}\right\}} \sum_{\left\{d_{l}\right\}}\left|\mathcal{N}^{(n)}\left(h_{i}, h_{j},\left\{h_{l}\right\}\right)\right|^{2}=\frac{4 \pi \alpha_{S}}{k_{i} \cdot k_{j}} P_{g g}^{<}(z)\left|\mathcal{A}^{(n-1)}\right|^{2},
$$

where

$$
\left|\mathcal{A}^{(n-1)}\right|^{2}=\sum_{h,\left\{h_{l}\right\}}\left|\mathcal{A}^{(n-1)}\left(h,\left\{h_{l}\right\}\right)\right|^{2}
$$

is the full $(n-1)$-particle transition amplitude squared and summed over helicities and colors. From it, we define the Born amplitude squared by multiplying by the flux factor and averaging over the spin and color of the incoming particles

$$
\mathcal{M}^{(n-1)}=\frac{1}{2 k_{1} \cdot k_{2}} \frac{1}{\omega\left(a_{1}\right) \omega\left(a_{2}\right)}\left|\mathcal{A}^{(n-1)}\right|^{2} .
$$

When considering three-jet production at the next-to-leading order, this quantity coincides with $\mathcal{M}^{(3,0)}$, defined in eq. (2.10). Eq. (B.13) can be simplified using the explicit form of the splitting functions with fixed helicities. We have

$$
S_{g g}^{+h_{i} h_{j}}(z)\left(S_{g g}^{-h_{i} h_{j}}(z)\right)^{*}=\delta_{h_{i} \bar{h}_{j}} \frac{z(1-z)}{[i j]^{2}} .
$$

Therefore we get

$$
\begin{aligned}
\sum_{h_{i}, h_{j},\left\{h_{l}\right\}} & \sum_{\left\{d_{l}\right\}} \mathcal{R}\left(h_{i}, h_{j},\left\{h_{l}\right\}\right)=32 \pi \alpha_{S} C_{A} z(1-z) \\
& \times \operatorname{Re}\left\{\frac{1}{[i j]^{2}} \sum_{\left\{h_{l}\right\}} \sum_{d_{e},\left\{d_{l}\right\}} \mathcal{A}_{d_{e}}^{(n-1)}\left(+,\left\{h_{l}\right\}\right)\left(\mathcal{A}_{d_{e}}^{(n-1)}\left(-,\left\{h_{l}\right\}\right)\right)^{*}\right\} .
\end{aligned}
$$

We can now write the full $n$-particle amplitude squared

$$
\mathcal{M}^{(n)}=\frac{1}{2 k_{1} \cdot k_{2}} \frac{1}{\omega\left(a_{1}\right) \omega\left(a_{2}\right)}\left|\mathcal{A}^{(n)}\right|^{2} .
$$

For three-jet production at the next-to-leading order, $\mathcal{M}^{(n)}$ coincides with $\mathcal{M}^{(4)}$, defined in eq. (2.18). Using eqs. (B.16), (B.18) and (B.20) we get

$$
\mathcal{M}^{(n)} \stackrel{i \| j}{\longrightarrow} \frac{4 \pi \alpha_{S}}{k_{i} \cdot k_{j}} P_{g g}^{<}(z) \mathcal{M}^{(n-1)}-\frac{16 \pi \alpha_{S}}{k_{i} \cdot k_{j}} C_{A} z(1-z) \tilde{\mathcal{M}}^{(n-1)},
$$


where

$$
\begin{aligned}
\tilde{\mathcal{M}}^{(n-1)} & =\frac{1}{2 k_{1} \cdot k_{2}} \frac{1}{\omega\left(a_{1}\right) \omega\left(a_{2}\right)} \\
& \times \operatorname{Re}\left\{\frac{<i j>}{[i j]} \sum_{\left\{h_{l}\right\}} \sum_{d_{e},\left\{d_{l}\right\}} \mathcal{A}_{d_{e}}^{(n-1)}\left(+,\left\{h_{l}\right\}\right)\left(\mathcal{A}_{d_{e}}^{(n-1)}\left(-,\left\{h_{l}\right\}\right)\right)^{*}\right\} .
\end{aligned}
$$

Notice that, apart from the factor $<i j>/[i j], \tilde{\mathcal{M}}^{(n-1)}$ would be equal to the Born amplitude squared, eq. (B.18), if the first entry of $\mathcal{A}^{(n-1)}$ were equal to the first entry of $\left(\mathcal{A}^{(n-1)}\right)^{*}$. In eqs. $(\mathbb{B} .18),(\mathbb{B} .21)-(\mathbb{B} .23)$ we suppressed the obvious functional dependence, but we emphasize that, while $\mathcal{M}^{(n-1)}$ is independent from $k_{i}$ and $k_{j}, \tilde{\mathcal{M}}^{(n-1)}$ has a residual dependence upon these momenta through the factor $<i j>/[i j]$, which explicitly appears in eq. (B.23).

We now turn to case b), that is the splitting $g \rightarrow q \bar{q}$. We use again eq. (B.6) to define the $z$ parameter, and assign color indices $k$ and $\bar{k}$ to the quark $i$ and to the antiquark $j$ respectively. Eq. (B.7) still holds, with the formal substitution

$$
C\left(d_{e}, b, c\right) \rightarrow C\left(d_{e}, k, \bar{k}\right) \equiv\left(\lambda^{d_{e}}\right)_{k \bar{k}}
$$

Obviously, we have also to substitute everywhere $P_{g g}^{<}$with $P_{q g}^{<}$. Instead of eq. (B.9) we have

$$
\sum_{k, k^{\prime}} \sum_{\bar{k}, \bar{k}^{\prime}} \delta_{k k^{\prime}} \delta_{\bar{k} \bar{k}^{\prime}} C\left(d_{e}, k, \bar{k}\right) C^{*}\left(d_{e}^{\prime}, k^{\prime}, \bar{k}^{\prime}\right)=2 T_{F} \delta_{d_{e} d_{e}^{\prime}}
$$

The derivation goes unchanged from this point on. We have only to take into account that

$$
\sum_{h_{i}, h_{j}}\left|S_{q g}^{+h_{i} h_{j}}(z)\right|^{2}=\sum_{h_{i}, h_{j}}\left|S_{q g}^{-h_{i} h_{j}}(z)\right|^{2}=\frac{1}{T_{F}} \frac{1}{2 k_{i} \cdot k_{j}} P_{q g}^{<}(z),
$$

and

$$
S_{q g}^{+h_{i} h_{j}}(z)\left(S_{q g}^{-h_{i} h_{j}}(z)\right)^{*}=-\delta_{h_{i} \bar{h}_{j}} \frac{z(1-z)}{[i j]^{2}} .
$$

Eq. (B.22) gets therefore modified as follows

$$
\mathcal{M}^{(n)} \stackrel{i \| j}{\longrightarrow} \frac{4 \pi \alpha_{S}}{k_{i} \cdot k_{j}} P_{q g}^{<}(z) \mathcal{M}^{(n-1)}+\frac{16 \pi \alpha_{S}}{k_{i} \cdot k_{j}} T_{F} z(1-z) \tilde{\mathcal{M}}^{(n-1)} .
$$

Finally, we deal with the splitting $q \rightarrow q g$. With obvious modifications due to the fact that the exchanged particle in now a quark instead of a gluon, we can follow the derivation outlined before. Nevertheless, we immediately understand that in this case there is no $\mathcal{R}$ term. In fact, we know from eq. (B.10) that such a term is present if and 
only if for a given external helicity configuration the exchanged virtual particle can have both helicities. In the present case, this is forbidden due to helicity conservation along the quark line. More formally, for the $q \rightarrow q g$ splitting we have always

$$
S_{g q}^{+h_{i} h_{j}}(z)\left(S_{g q}^{-h_{i} h_{j}}(z)\right)^{*}=S_{q q}^{+h_{i} h_{j}}(z)\left(S_{q q}^{-h_{i} h_{j}}(z)\right)^{*}=0
$$

for every possible choice of $h_{i}$ and $h_{j}$.

We collect now the results obtained so far, also explicitly indicating the dependence upon the momenta of the particles, referring to eq. (B.6) for the definition of the kinematics:

$$
\begin{aligned}
\mathcal{M}^{(n)}\left(k_{1}, k_{2} ; . ., k_{i}, . ., k_{j}, . .\right) & \stackrel{i \| j}{\longrightarrow} \\
& \frac{4 \pi \alpha_{S}}{k_{i} \cdot k_{j}} P_{a_{i} S\left(a_{i}, a_{j}\right)}^{<}(z) \mathcal{M}^{(n-1)}\left(k_{1}, k_{2} ; . ., k_{P}, . .\right) \\
& +\frac{4 \pi \alpha_{S}}{k_{i} \cdot k_{j}} Q_{a_{i} S\left(a_{i}, a_{j}\right)^{\star}}(z) \tilde{\mathcal{M}}^{(n-1)}\left(k_{1}, k_{2} ; . ., k_{i}, . ., k_{j}, . .\right),
\end{aligned}
$$

where

$$
\begin{aligned}
Q_{g g^{\star}}(z) & =-4 C_{A} z(1-z), \\
Q_{q g^{\star}}(z) & =4 T_{F} z(1-z), \\
Q_{g q^{\star}}(z) & =0 \\
Q_{q q^{\star}} & =0
\end{aligned}
$$

In these equations, the ${ }^{\star}$ symbol over the flavour of the particle that eventually splits reminds that this particle is off-shell. In principle, this notation should be extended also to the Altarelli-Parisi splitting kernels, but at the leading order $P_{a b^{\star}}=P_{a^{\star} b}$, and therefore there is no need to keep track of the off-shell particle. By construction, the $\Delta$ term of section 4 is equal to $Q \tilde{\mathcal{M}}^{(n-1)}$.

We have now to deal with the collinear emission from an incoming particle. We have two options: we can perform an explicit calculation, as we have done for the final state emission; or otherwise we can exploit the crossing symmetry property of the $n$-particle amplitude [19]. We will pursue this second option, and to be definite we will restrict to emission from particle 1 . To proceed further, we have to think in terms of an (unphysical) amplitude, for which also the particle 1 is outgoing; the 
limit $1 \| j$ will therefore be given by eq. (B.30) (with obvious modifications in the notation). The kinematics will be given by eq. (B.6)

$$
\bar{k}_{1}=z k_{P}, \quad k_{j}=(1-z) k_{P},
$$

where $\bar{k}_{1}=-k_{1}$ since in the unphysical amplitude the particle 1 has negative energy. In doing the crossing, apart from the substitution $\bar{k}_{1} \rightarrow k_{1}$, we will have to refer the kinematics to $k_{1}$, that is

$$
k_{j}=(1-z) k_{1}, \quad k_{P}=z k_{1}
$$

or

$$
z \longrightarrow 1 / z
$$

Since $k_{P}$ is the four-momentum of an incoming particle in the $(n-1)$-particle amplitude, the second equation in (B.36) implies that the flux factor of the $(n-1)$-particle amplitude will be $1 / z$ times the flux factor of the $n$-particle amplitude. Therefore, to have the correct normalization, we have to multiply by $z$ the $(n-1)$-particle amplitude. Furthermore, since the flavour of 1 and $P$ can be different, a factor $\omega\left(a_{P}\right) / \omega\left(a_{1}\right)$ will take into account the different color and spin normalization factors of the $n$-particle and $(n-1)$-particle amplitudes. Eq. (B.30) becomes therefore

$$
\begin{aligned}
& \mathcal{M}^{(n)}\left(k_{1}, k_{2} ; . ., k_{j}, . .\right) \stackrel{1 \| j}{\longrightarrow} \\
& -(-)^{\left[\sigma\left(a_{1}\right)+\sigma\left(S\left(a_{1}, \bar{a}_{j}\right)\right]\right.} \frac{4 \pi \alpha_{S}}{k_{1} \cdot k_{j}} \frac{\omega\left(S\left(a_{1}, \bar{a}_{j}\right)\right)}{\omega\left(a_{1}\right)} z P_{\bar{a}_{1} S\left(\bar{a}_{1}, a_{j}\right)}^{<}\left(\frac{1}{z}\right) \mathcal{M}^{(n-1)}\left(z k_{1}, k_{2} ; . .\right) \\
& -(-)^{\left[\sigma\left(a_{1}\right)+\sigma\left(S\left(a_{1}, \bar{a}_{j}\right)\right]\right.} \frac{4 \pi \alpha_{S}}{k_{1} \cdot k_{j}} \frac{\omega\left(S\left(a_{1}, \bar{a}_{j}\right)\right)}{\omega\left(a_{1}\right)} z Q_{\bar{a}_{1} S\left(\bar{a}_{1}, a_{j}\right)^{\star}}\left(\frac{1}{z}\right) \tilde{\mathcal{M}}^{(n-1)}\left(z k_{1}, k_{2} ; . ., k_{j}, . .\right),
\end{aligned}
$$

where $\sigma(g)=0$ and $\sigma(q)=1$ (therefore, $(-)^{\sigma}$ takes into account the crossing of a fermionic line), and the overall minus sign is due to the $\bar{k}_{1} \rightarrow k_{1}$ substitution. We can now exploit the crossing symmetry property of the Altarelli-Parisi kernels (for $z<1$ )

$$
P_{b a}^{<}(z)=-(-)^{[\sigma(a)+\sigma(b)]} \frac{\omega(b)}{\omega(a)} z P_{\bar{a} \bar{b}}^{<}\left(\frac{1}{z}\right) .
$$

For consistency with this equation, we are thus led to define (notice the ${ }^{\star}$ symbol)

$$
Q_{b^{\star} a}(z)=-(-)^{[\sigma(a)+\sigma(b)]} \frac{\omega(b)}{\omega(a)} z Q_{\bar{a} \bar{b}^{\star}}\left(\frac{1}{z}\right) .
$$


Eq. (B.38) becomes therefore

$$
\begin{aligned}
\mathcal{M}^{(n)}\left(k_{1}, k_{2} ; . ., k_{j}, . .\right) & \stackrel{1 \| j}{\longrightarrow} \frac{4 \pi \alpha_{S}}{k_{1} \cdot k_{j}} P_{S\left(a_{1}, \bar{a}_{j}\right) a_{1}}^{<}(z) \mathcal{M}^{(n-1)}\left(z k_{1}, k_{2} ; . .\right) \\
& +\frac{4 \pi \alpha_{S}}{k_{1} \cdot k_{j}} Q_{S\left(a_{1}, \bar{a}_{j}\right)^{\star} a_{1}}(z) \tilde{\mathcal{M}}^{(n-1)}\left(z k_{1}, k_{2} ; . ., k_{j}, . .\right),(B . .)
\end{aligned}
$$

where, from the definition in eq. (B.40), and using eqs. (B.31)-(B.34),

$$
\begin{aligned}
& Q_{g^{\star} g}(z)=-4 C_{A} \frac{1-z}{z}, \\
& Q_{q^{\star} g}(z)=0, \\
& Q_{g^{\star} q}(z)=-4 C_{F} \frac{1-z}{z}, \\
& Q_{q^{\star} q}(z)=0 .
\end{aligned}
$$

In spite of the fact that the $\Delta$ term has no deep physical meaning, still the crossing symmetry property has to hold true, and the relation between $Q_{a b^{\star}}$ and $Q_{b^{\star} a}$ should not be regarded as purely incidental, that is, due to the definition (B.40). In fact, directly performing the calculation for the initial state collinear splitting, and defining $Q_{b^{\star} a}$ through eq. (B.41), we obtain again eqs. (B.42)-(B.45).

We finally list the results for $\tilde{\mathcal{M}}^{(5)}$ for processes in which only massless partons are involved. This kind of processes are relevant for three-jet production. The corresponding Born amplitudes squared, $\mathcal{M}^{(5)}$, are well known from the literature (see for example ref. [30]).

We start with the purely gluonic processes, denoting the momenta of the gluons which do not split by $k, l, m, n$, and with $P$ the momentum of the gluon which eventually splits. We get

$$
\begin{aligned}
& \tilde{\mathcal{M}}^{(5 g)}= \frac{1}{2 k_{1} \cdot k_{2}} \frac{4 g_{S}^{6} N_{c}^{3}\left(N_{c}^{2}-1\right)}{\omega\left(a_{1}\right) \omega\left(a_{2}\right)} \\
& \times \operatorname{Re}\left\{\frac{\langle i j\rangle}{[i j]}\left(\langle k l\rangle^{4}\langle m n\rangle^{4}+\langle k m\rangle^{4}\langle l n\rangle^{4}+\langle k n\rangle^{4}\langle l m\rangle^{4}\right)\right. \\
&\left.\quad \times \sum_{\sigma^{*}(k, l, m, n)}(\langle k l\rangle\langle l m\rangle\langle m n\rangle\langle n P\rangle\langle P k\rangle)^{-2}\right\} .
\end{aligned}
$$

Here $\sigma^{*}(k, l, m, n)$ denotes all the permutations of the elements $k, l, m, n$ which are inequivalent under reflection $(\{k l m n\} \rightarrow\{n m l k\})$. For the processes with two quarks 
and three gluons we denote by $m$ and $n$ the momenta of the gluons which do not participate in the splitting, and by $q$ and $\bar{q}$ the momenta of the quark-antiquark pair. We have

$$
\begin{aligned}
\tilde{\mathcal{M}}^{(2 q 3 g)}= & -\frac{1}{2 k_{1} \cdot k_{2}} \frac{4 g_{S}^{6}}{\omega\left(a_{1}\right) \omega\left(a_{2}\right)} \\
\times & \operatorname{Re}\left\{\frac{\langle i j\rangle}{[i j]} \frac{\langle q m\rangle\langle\bar{q} m\rangle\langle\bar{q} n\rangle\langle q n\rangle}{\langle\bar{q} q\rangle^{2}}\left(\langle\bar{q} m\rangle^{2}\langle q n\rangle^{2}+\langle q m\rangle^{2}\langle\bar{q} n\rangle^{2}\right)\right. \\
& \times\left[\frac{\left(N_{c}^{2}-1\right)^{3}}{2 N_{c}^{2}} \sum_{\sigma(m, n, P)}(\langle\bar{q} m\rangle\langle m n\rangle\langle n P\rangle\langle P q\rangle)^{-2}\right. \\
& +\frac{\left(N_{c}^{2}-1\right)^{2}}{N_{c}^{2}} \sum_{\sigma^{*}(m, n, P)}\left(\frac{1}{\langle\bar{q} m\rangle\langle m n\rangle\langle n P\rangle\langle P \bar{q}\rangle\langle q n\rangle^{2}\langle m P\rangle^{2}}+(q \leftrightarrow \bar{q})\right) \\
& \left.\left.+\left(N_{c}^{2}-1\right) \sum_{\sigma^{*}(m, n, P)} \frac{1}{\langle\bar{q} m\rangle\langle m q\rangle\langle q P\rangle\langle P \bar{q}\rangle\langle m n\rangle^{2}\langle P n\rangle^{2}}\right]\right\} .
\end{aligned}
$$

Finally, for processes with four quarks and one gluon, we have to distinguish between the case in which there are two quark-antiquark pairs of different flavour, and the case in which the flavour of the two pairs is the same. In both cases, we denote the momenta of the quarks by $q, \bar{q}, Q$ and $\bar{Q}$. The momentum of the splitting gluon is again $P$. For quark-antiquark pairs of different flavour we get

$$
\begin{aligned}
\tilde{\mathcal{M}}_{\mathrm{DF}}^{(4 q 1 g)}= & \frac{1}{2 k_{1} \cdot k_{2}} \frac{2 g_{S}^{6}}{\omega\left(a_{1}\right) \omega\left(a_{2}\right)} \operatorname{Re}\left\{\frac{\langle i j\rangle}{[i j]} \frac{\langle\bar{q} \bar{Q}\rangle^{2}\langle q Q\rangle^{2}+\langle\bar{q} Q\rangle^{2}\langle q \bar{Q}\rangle^{2}}{\langle\bar{q} q\rangle^{2}\langle\bar{Q} Q\rangle^{2}}\right. \\
& \times\left[\left(N_{c}^{3}-N_{c}\right)\left(\frac{\langle\bar{q} Q\rangle^{2}}{\langle Q P\rangle^{2}\langle\bar{q} P\rangle^{2}}+\frac{\langle q \bar{Q}\rangle^{2}}{\langle q P\rangle^{2}\langle\bar{Q} P\rangle^{2}}\right)\right. \\
& +\frac{N_{c}^{2}-1}{N_{c}}\left(\frac{\langle\bar{q} q\rangle^{2}}{\langle\bar{q} P\rangle^{2}\langle q P\rangle^{2}}-\frac{2\langle\bar{q} q\rangle\langle\bar{q} Q\rangle}{\langle\bar{q} P\rangle^{2}\langle q P\rangle\langle Q P\rangle}-\frac{2\langle q \bar{q}\rangle\langle q \bar{Q}\rangle}{\langle q P\rangle^{2}\langle\bar{q} P\rangle\langle\bar{Q} P\rangle}\right. \\
& \left.\left.\left.+\frac{\langle\bar{Q} Q\rangle^{2}}{\langle\bar{Q} P\rangle^{2}\langle Q P\rangle^{2}}-\frac{2\langle q \bar{Q}\rangle\langle Q \bar{Q}\rangle}{\langle\bar{Q} P\rangle^{2}\langle q P\rangle\langle Q P\rangle}-\frac{2\langle\bar{q} Q\rangle\langle\bar{Q} Q\rangle}{\langle Q P\rangle^{2}\langle\bar{q} P\rangle\langle\bar{Q} P\rangle}\right)\right]\right\} .
\end{aligned}
$$


For quark-antiquark pairs of equal flavour we have

$$
\begin{aligned}
\tilde{\mathcal{M}}_{\mathrm{EF}}^{(4 q 1 g)}= & \tilde{\mathcal{M}}_{\mathrm{DF}}^{(4 q 1 g)}+\tilde{\mathcal{M}}_{\mathrm{DF}}^{(4 q 1 g)}(q \leftrightarrow Q) \\
- & \frac{1}{2 k_{1} \cdot k_{2}} \frac{4 g_{S}^{6}\left(N_{c}^{2}-1\right)}{\omega\left(a_{1}\right) \omega\left(a_{2}\right)} \operatorname{Re}\left\{\frac{\langle i j\rangle}{[i j]} \frac{\langle\bar{q} \bar{Q}\rangle^{2}\langle q Q\rangle^{2}}{\langle\bar{q} q\rangle\langle\bar{q} Q\rangle\langle q \bar{Q}\rangle\langle\bar{Q} Q\rangle}\right. \\
& \times\left[\frac{\langle\bar{q} q\rangle^{2}}{\langle\bar{q} P\rangle^{2}\langle q P\rangle^{2}}+\frac{\langle\bar{q} Q\rangle^{2}}{\langle\bar{q} P\rangle^{2}\langle Q P\rangle^{2}}+\frac{\langle\bar{Q} q\rangle^{2}}{\langle\bar{Q} P\rangle^{2}\langle q P\rangle^{2}}+\frac{\langle\bar{Q} Q\rangle^{2}}{\langle\bar{Q} P\rangle^{2}\langle Q P\rangle^{2}}\right. \\
& -\frac{N_{c}^{2}+1}{N_{c}^{2}}\left(\frac{\langle\bar{q} q\rangle\langle\bar{q} Q\rangle}{\langle\bar{q} P\rangle^{2}\langle q P\rangle\langle Q P\rangle}+\frac{\langle\bar{Q} q\rangle\langle\bar{Q} Q\rangle}{\langle\bar{Q} P\rangle^{2}\langle q P\rangle\langle Q P\rangle}\right. \\
& \left.\left.\left.\quad+\frac{\langle q \bar{q}\rangle\langle q \bar{Q}\rangle}{\langle q P\rangle^{2}\langle\bar{q} P\rangle\langle\bar{Q} P\rangle}+\frac{\langle Q \bar{q}\rangle\langle Q \bar{Q}\rangle}{\langle Q P\rangle^{2}\langle\bar{q} P\rangle\langle\bar{Q} P\rangle}\right)\right]\right\}
\end{aligned}
$$

\section{References}

[1] For recent reviews see:

J. E. Huth and M. L. Mangano, Ann. Rev. Nucl. Part. Sc. 42(1992)251;

R. K. Ellis and W. J. Stirling, Fermilab-Conf-90/164-T (1990) (unpublished).

[2] S. D. Ellis, Z. Kunszt and D. E. Soper, Phys. Rev. Lett. 62(1988)726;

Phys. Rev. Lett. 64(1990)2121;

F. Aversa, M. Greco, P. Chiappetta and J. P. Guillet, Phys. Lett. B210(1988)225;

Phys. Lett. B211(1988)465; Nucl. Phys. B327(1989)105; Z. Phys. C46(1990)253;

Phys. Rev. Lett. 65(1990)401.

[3] D. Bödeker, G. Kramer, S. G. Salesh, Z. Phys. C63(1994)471;

J. R. Forshaw and R. G. Roberts, Phys. Lett. B348(1995)665;

L. E. Gordon and J. K. Storrow, Phys. Lett. B319(1993)539;

M. Klasen and G. Kramer, preprint DESY-95-159, hep-ph/9508337.

[4] S. D. Ellis, Z. Kunszt and D. E. Soper, Phys. Rev. Lett. 69(1992)1496;

W. T. Giele, E. W. N. Glover and D. A. Kosower, Phys. Rev. Lett. 73(1994)2019;

S. D. Ellis and D. E. Soper, Phys. Rev. Lett. 74(1995)5182.

[5] CDF Collaboration, F. Abe et al., Phys. Rev. D45(1992)1448.

[6] CDF Collaboration, F. Abe et al., Phys. Rev. Lett. 68(1992)1104;

Phys. Rev. Lett. 70(1993)1376. 
[7] ZEUS Collaboration, M. Derrick et al., Phys. Lett. B342(1995)417;

Phys. Lett. B348(1995)665.

[8] H1 Collaboration, I. Abt et al., Phys. Lett. B314(1993)436.

[9] CDF Collaboration, F. Abe et al., Nucl. Phys. B269(1986)445;

Phys. Rev. D41(1990)1722; Phys. Rev. Lett. 68(1992)1104;

D0 Collaboration, F. Nang et al., Fermilab Report

No. FERMILAB-Conf-94/323-E (1994).

[10] J. Huston et al., preprint CTEQ-512 (November, 1995).

[11] UA1 Collaboration, G. Arnison et al., Phys. Lett. B158(1985)494;

UA2 Collaboration, J. A. Appel et al., Z. Phys. C30(1986)341;

CDF Collaboration, F. Abe et al., Phys. Rev. D45(1992)1448;

Phys. Rev. Lett. 75(1995)608;

D0 Collaboration, S. Abachi et al., hep-ph/9508337;

[12] Z. Bern, L. Dixon and D. A. Kosower, Phys. Rev. Lett. 70(1993)2677.

[13] Z. Kunszt, A. Signer and Z. Trócsányi, Phys. Lett. B336(1994)529.

[14] Z. Bern, L. Dixon and D. A. Kosower, Nucl. Phys. B437(1995)259.

[15] A. Signer, PhD Thesis, ETH-Zurich, 1995.

[16] J. Gunion and Z. Kunszt, Phys. Lett. 159B(1985)167; Phys. Lett. 161B(1985)333;

Phys. Lett. 176B(1985)163;

Z. Kunszt, Nucl. Phys. B271(1986)333;

S. J. Parke and T. R. Taylor, Nucl. Phys. B269(1986)410;

M. L. Mangano, S. J. Parke and Z. Xu, Nucl. Phys. B298(1988)653;

J. Gunion and J. Kalinowski, Phys. Rev. D34(1986)2119;

F. A. Berends and W. T. Giele, Nucl. Phys. B306(1988)759.

[17] J. G. M. Kuijf, PhD thesis, Leiden 1991.

[18] M. L. Mangano and S. J. Parke, Phys. Rep. 200(1991)301.

[19] Z. Kunszt and D. E. Soper, Phys. Rev. D46(1992)192.

[20] S. D. Ellis, Z. Kunszt and D. E. Soper, Phys. Rev. D40(1989)2188. 
[21] W. T. Giele and E. W. N. Glover, Phys. Rev. D46(1992)1980;

W. T. Giele, E. W. N. Glover and D. A. Kosower, Nucl. Phys. B403(1993)633.

[22] B. Mele, P. Nason and G. Ridolfi, Nucl. Phys. B357(1991)409;

M. Mangano, P. Nason and G. Ridolfi, Nucl. Phys. B373(1992)295;

S. Frixione, P. Nason and G. Ridolfi, Nucl. Phys. B383(1992)3;

S. Frixione, Nucl. Phys. B410(1993)280;

S. Frixione, M. Mangano, P. Nason and G. Ridolfi, Nucl. Phys. B412(1994)225.

[23] J. C. Collins, D. E. Soper and G. Sterman, in Perturbative Quantum Chromodinamics, 1989, ed. Mueller, World Scientific, Singapore, and references therein.

[24] S. D. Ellis and D. E. Soper, Phys. Rev. D48(1993)3160;

see also: S. Catani, Yu. Dokshitser, M. A. Seymour and B. R. Webber, Nucl. Phys. B406(1993)187.

[25] D0 Collaboration, proceedings of the $8^{\text {th }}$ meeting of the American Physical Society, Albuquerque, NM, August $2^{\text {nd }}-6^{\text {th }}, 1994$.

[26] F. Aversa et al., Proceedings of the Summer Study on High Energy Physics, Research Directions for the Decade, Snowmass, CO, Jun 25 - Jul 13, 1990.

[27] Z. Kunszt, A. Signer and Z. Trócsányi, Nucl. Phys. B420(1994)550.

[28] R. K. Ellis and J. Sexton, Nucl. Phys. B269(1986)445.

[29] Z. Kunszt, A. Signer and Z. Trócsányi, Nucl. Phys. B411(1994)397.

[30] F. A. Berends et al., Phys. Lett. B103(1981)124. 